\title{
Development of an Effective Non-Linear Normal-Mode Initialization Method for a High-Resolution Global Model
}

\author{
Hiroyuki MURAKAMI \\ Advanced Earth Science and Technology Organization (AESTO), Japan Meteorological Agency, Tokyo, Japan \\ and \\ Takayuki MATSUMURA ${ }^{1}$ \\ Numerical Prediction Division, Japan Meteorological Agency, Tokyo, Japan \\ (Manuscript received 24 July 2006, in final form 21 December 2006)
}

\begin{abstract}
An efficient non-linear normal-mode initialization (NNMI) scheme for a super high-resolution atmospheric general circulation model (AGCM) has been developed. The conventional NNMI method, based on Machenhauer (1977), is difficult to apply to such an AGCM, with a grid spacing of $20 \mathrm{~km}$, since it demands an extremely large computational memory. The new scheme combines two techniques; one is the vertical-mode initialization method proposed by Bourke and McGregor (1983), and the other is the incremental NNMI method proposed by Ballish et al. (1992). Comparisons with the conventional Machenhauer scheme, using a $60 \mathrm{~km}$-resolution AGCM, show that the new scheme favorably suppresses undesirable gravity-wave oscillations, while maintaining meaningful tidal waves in the initial states. A medium-range forecast experiment with data assimilation shows that the new scheme is also competitive with the conventional scheme in terms of forecast skill. It is demonstrated that the new scheme is efficient and effective for a $20 \mathrm{~km}$-resolution AGCM.
\end{abstract}

\section{Introduction}

A time-slice experiment method (Bengtsson et al. 1996; IPCC 2001), in which a highresolution atmospheric general circulation model (AGCM) is forced by sea surface temperature from a coupled ocean-atmosphere model, is a vital ingredient for a global warming projection to assess how socio-economic impacts of

Corresponding author and present affiliation: Hiroyuki Murakami, Center for Environmental Information Science (CEIS), Ponpian-building, 8-19, Yonban-cho, Chiyoda-ku, Tokyo 102-0081, Japan. E-mail: hir.murakami@gmail.com.

1. Present affiliation: Sapporo District Meteorological Observatory, Sapporo, Japan

(C) 2007, Meteorological Society of Japan hazardous weather, such as tropical cyclones, change due to global warming. The Advanced Earth Science and Technology Organization (AESTO), the Meteorological Research Institute (MRI), and the Numerical Prediction Division of the Japan Meteorological Agency (JMA) have developed a super high-resolution (TL959L60; horizontal $20 \mathrm{~km}$-mesh, vertical 60 layers) AGCM (JMA/MRI-AGCM) (Mizuta et al. 2006) to investigate the effect of global warming on typhoons (Oouchi et al. 2006) and Baiu (Kusunoki et al. 2006) under the Intergovernmental Panel on Climate Change (IPCC) scenarios. The time-slice experiments are performed on the Earth Simulator (ES) (Habata et al. 2004).

Oouchi et al. (2006) showed that the number 
of strong tropical cyclones increases while the occurrence of tropical cyclones is reduced globally in a warm-climate environment. They carefully evaluated the reliability of their projection of tropical cyclones by comparing their presentday 10-year experiment with observations with respect to geographical distribution, frequency, and intensity. In order to obtain further reliability, it is also necessary to evaluate simulations for an individual tropical cyclone, in terms of its genesis, track, and intensity through medium-range forecast experiments with data assimilation.

A medium-range forecast experiment is an initial condition problem; thus, an initialization procedure is necessary to reduce spurious noise in the initial state, brought mainly by the transformation of resolution, for the initial data. Although the JMA/MRI-AGCM is originally furnished with a module of a non-linear normal mode initialization (NNMI), applying it to a super high-resolution model, with a grid spacing of $20 \mathrm{~km}$, requires several challenges.

The NNMI was developed after Machenhauer (1977) and Baer (1977) independently applied it to shallow water equations. After their studies, the NNMI was applied to global primitive equation models by Daley (1979), Temperton and Williamson (1981), and Williamson and Temperton (1981). The NNMI is a technique to reduce spurious imbalances between mass and wind fields introduced by an analysis. Procedures for deriving each normal mode are thoroughly reviewed in Daley (1981) and Daley (1991). The NNMI has been widely used, because it can distinguish gravity modes and successfully reduce gravity noise. The essence of the Machenhauer NNMI (MNMI) is to suppress spurious gravity waves by setting the time derivative of the fast gravity modes to zero.

However, the MNMI has two disadvantages. One is that the MNMI also removes meaningful gravity modes, such as tidal waves, from initial data, unless fast modes whose eigen-periods are shorter than 12 hours are excluded from the procedure (Kudo 1982; Wergen 1987; Ballish et al. 1992). In order to prevent the removal of tidal waves, the original method of the JMA/ MRI-AGCM adds climatological tidal modes, created by the model, to the initialized data (JMA 2002). Although introducing the climato- logical tidal modes is fairly practical, it is recognized that the MNMI with the climatological tidal modes induces a somewhat unnatural modification pattern (to be described in Section 4.b).

The other disadvantage is the computational cost of memory allocation for horizontal discretization. Daley (1988) has shown that the cost becomes much larger as the horizontal resolution becomes higher in the MNMI (to be described in Section 3.c). As an alternative, a dynamic filtering technique, such as a digital filter, has recently become popular. The digital filtering technique was first applied to numerical weather prediction by Lynch and Huang (1992), and later extended to a diabatic process by Huang and Lynch (1993). The filtering technique is designed to remove high-frequency noise from a time series, which is produced through a time integration of a model. A considerable advantage of the digital filter over the NNMI method is its simplicity of implementation for both adiabatic and full-physics models (Huang and Lynch 1993; Huang and Sundqvist 1993; Huang et al. 1994; Polavarapu et al. 2000). Since the digital filter does not require a complex procedure for determining a Rossby or gravity-mode structure, the digital filter has been rapidly replacing the NNMI as an initialization scheme (Fillion et al. 1995; Lynch et al. 1997; Gauthier and Thépaut 2001). However, the digital filter also seems to have the same problem as the MNMI, namely, vast computational resources are required in a super highresolution case, because the digital filter requires integration both forward and backward for a few hours in the filtering process.

Recently, in addition to the refining model resolution, an analysis method of fourdimensional variational assimilation (4DVAR) is widely used, and it provides stable initial condition which is consistent with model dynamics. Although the demand of the initialization seems to be small because stable analysis is created by the $4 \mathrm{DVAR}$, it is still possible that analysis field contains spurious high-frequency noise. For example, a low-resolution model is often used for minimizing the cost function in the 4DVAR. When the low-resolution analysis increment is added to the original-resolution first guess, the resolution difference is thought to cause the noise. (e.g., The noise may be 
mainly caused by boundary condition difference in the topography.) Courtier and Talagrand (1990) and Polavarapu et al. (2000) also indicated that if the observations have errors, analysis with the 4DVAR will still contain spurious high-frequency noise. Therefore, initialization is still necessary, even for the 4DVAR analysis scheme.

In this paper, an efficient NNMI method for a super high-resolution global model is presented as a preparation for the verification of tropical cyclone forecasts. The presented initialization scheme consists of two methods; one is the vertical normal-mode initialization (VNMI) proposed by Bourke and McGregor (1983), and the other is the incremental normal-mode initialization (INMI) proposed by Ballish et al. (1992). The effectiveness of the above NNMI method for a high-resolution model is mainly led by the VNMI, since the VNMI does not require explicit identification of the horizontal normal modes. In the VNMI theory, the $\beta$ terms (the meridional derivative of the Coriolis parameter) are set to zero by an $f$-plane approximation. When the $\beta$ terms are omitted, a horizontal normal mode is determined given only all wavenumbers. The $f$ plane approximation is usually applied to limited area models. In this study, however, the VNMI is applied to a global model together with the INMI. This relevance will be described again in Section 3.c in detail.

This paper is basically for an application of the non-linear normal mode initialization method with a hydrostatic, spectral, and anelastic AGCM. Hence, it requires gravity normal modes are explicitly determined. Furthermore, it requires there are no acoustic modes of oscillations. However, when the horizontal resolution becomes $10 \mathrm{~km}$-mesh or finer, nonhydrostatic effect becomes important. For such a finer model, the hydrostatic approximation is no longer appropriate. Although that makes it difficult to determine normal modes in a nonhydrostatic model, Daley (1988) and Kasahara and Qian (2000) showed normal modes of nonhydrostatic global model can be explicitly determined with some approximations. In the cases, our new initialization scheme is also applicable to control the growth of fast gravity waves. Although there also exists high frequency acoustic modes, which cause serious problem for in- tegration, Daley (1988) indicated they can be simply excised by using a filtering method proposed by Daley (1980).

This paper is organized as follows. A brief description of the JMA/MRI-AGCM, to which the new scheme is introduced, is presented in Section 2. Section 3 contains an explanation of the methodology of the MNMI and the new scheme. Section 4 displays some results of comparative experiments between the initialization schemes with a middle-resolution (TL319L40; horizontal $60 \mathrm{~km}$-mesh, vertical 40 layers) model. Section 4 also shows how to implement the new scheme with the super high-resolution model. Section 4 also shows a result of implementing the new scheme with the super highresolution model. A summary follows in Section 5.

\section{Description of JMA/MRI-AGCM}

The model, to which the new initialization scheme is introduced in this study, is the JMA/ MRI-AGCM. The model was developed through collaboration between the JMA, MRI, and AESTO for use of both climate simulations and weather predictions. The model has been used at the JMA for operational medium-range forecasts since February 17, 2005. The model has also been used for a global warming projection, with some modifications in physical processes (Kusunoki et al. 2006; Mizuta et al. 2006; Oouchi et al. 2006). Some of the outlines on the model are available in JMA (2002), and Mizuta et al. (2006). A simple description of the model is described here.

The model employs full primitive equations and a spectral transform method of spherical harmonics which were originally designed by Kanamitsu et al. (1983). It employs a sigmapressure hybrid coordinate as the vertical coordinate (Simmons and Burridge 1981). The model uses a new quasi-conservative semiLagrangian scheme (Yoshimura and Matsumura 2003) for stable and efficient time integration. The model includes a short-wave radiation scheme, which is important for tidal modes in the INMI. Short-wave scattering and absorption are modeled by a two-stream formulation using the delta-Eddington approximation (Joseph et al. 1976; Coakley et al. 1983), where the spectrum is divided into 18 bands, as described by Briegleb (1992). For the cumu- 
lus convection scheme, a prognostic Arakawa and Schubert (1974) scheme is implemented. Clouds are prognostically determined in a similar fashion to that of Smith (1990), in which a simple statistical approach, proposed by Sommeria and Deardorff (1977), is adopted for the calculation of the cloud amount and the cloud water content. The level 2 turbulence closure scheme of Mellor and Yamada (1974) is used to represent the vertical diffusion of momentum, heat, and moisture. The orographic gravity wave drag scheme is based on Palmer et al. (1986) and Wurtele et al. (1987), and thoroughly explained in Iwasaki et al. (1989). The Simple Biosphere scheme (SiB), developed by Sellers et al. (1986) and Sato et al. (1989), is implemented in the model. The model is originally provided with the MNMI, as described in Section 1. A stable iterative method (Kudo 1984) in the MNMI is introduced.

This model is well optimized for the ES (Katayama et al. 2003; Katayama et al. 2004). The computing efficiency with 60 nodes for the $20 \mathrm{~km}$-mesh JMA/MRI-AGCM is about $35 \%$ of the peak performance. In other words, the execution time of the 1-month simulation is about 3.5 hours, using 60 nodes of the ES.

\section{Initialization methodology}

In this section, the methodology of the NNMI is provided. First, the MNMI and an additional technique for treating tidal modes are described, and the description of the new initialization scheme then follows.

\section{a. Conventional Machenhauer's NNMI}

The MNMI (Machenhauer 1977) and an additional method of climatological tidal modes are the conventional initialization method for the JMA/MRI-AGCM. Their brief descriptions are available in JMA (2002).

In the MNMI process, an analysis field is filtered directly. First, discretization of vertical structure equations is carried out after linearizing the primitive equations using the atmosphere at rest as a basic state. In this study, the basic state has a constant temperature at each model level. Second, horizontal modes are derived for each vertical mode. The linearized parts of the equations are then projected onto the normal modes, which are divided into a set of high-frequent (fast) eastward, westward gravity modes, and a set of low-frequent (slow), Rossby modes.

When a fast gravity mode in an analysis field is described as $G_{A}$, the time tendency is described as the following equation:

$$
\frac{\partial G_{A}}{\partial t}=-i \omega G_{A}+N_{G_{A}},
$$

where the subscripted $A$ means the analysis field; $\omega$ represents the eigen-frequency of the gravity mode; and $N_{G_{A}}$ represents the nonlinear terms, which depend on all normal modes. The MNMI scheme requires that the time derivative of the modified fast gravity mode $G_{A}^{\prime}$ be set to zero,

$$
\frac{\partial G_{A}^{\prime}}{\partial t}=0 .
$$

Therefore, a modified gravity mode $G_{A}^{\prime}$ is given by the following equation,

$$
G_{A}^{\prime}=G_{A}+\frac{\partial G_{A} / \partial t}{i \omega}
$$

In the process, an iterative way is needed because non-linear terms depend upon all gravity modes. It can be solved by computing the time tendency by one-step time integration of the model from an analysis field, and deriving a new gravity mode by Eq. (3). Non-linear terms $N_{G_{A}}$ in Eq. (1) are also renewed. The MNMI is a kind of a method for adjusting non-linear terms in order not to grow fast-gravity modes. In a practical use, Kudo (1982) showed that modifying fast horizontal modes, with periods of less than 48 hours in five vertical modes, is enough to dampen erroneous noise.

However, Wergen (1987) warned that the filtering condition is inappropriate for atmospheric tides. The filtering condition of Eq. (2) is only appropriate if the forcing is quasistationary. This is clearly not the case if the radiation scheme simulates the diurnal cycle of the sun. The atmospheric tides of zonal wavenumber 1 and 2 are mostly excited by the absorption of solar radiation by ozone in the stratosphere and the mesosphere, and water vapor in the troposphere. Because of the vertical distribution of the absorbers, the diurnal zonal wavenumber 1 is largely trapped, whereas the semi-diurnal wave can propagate vertically (Chapman and Lindzen 1970). The fast 
gravity modes of periods less than 48 hours are modified by the filtering condition. However, this not only removes erroneous gravitational oscillations but also suppresses semi-diurnal tidal modes because the atmospheric tides are mostly projected onto gravity modes.

In order to avoid the problem discussed above, the time tendencies of climatological tidal modes are added after the time tendencies are set to zero. The filtering condition of Eq. (2) can be re-written namely as:

$$
\frac{\partial G_{A}^{\prime}}{\partial t}=\frac{\partial G_{T}}{\partial t},
$$

where $G_{T}$ is a climatological tidal mode. The modifying equation corresponding to Eq. (3) becomes

$$
G_{A}^{\prime}=G_{A}+\frac{\partial G_{A} / \partial t-\partial G_{T} / \partial t}{i \omega} .
$$

The time tendency of the climatological tidal modes can be obtained from a time series analysis by following Wergen (1987) as:

$$
\frac{\partial G_{T}}{\partial t}=\frac{1}{J} \sum_{j=1}^{J} \sum_{m=1}^{2} \frac{\partial G_{A}(j)}{\partial t} \exp \left(i \frac{\pi m}{2} j\right),
$$

where $j$ represents 6 -hour-apart entries in the time series; $J$ is the length of the time series; $G_{A}(j)$ is a gravity mode at $j$; and $m$ is a zonal wavenumber. The zonal wavenumber 1 and 2 correspond to the 24-hour and 12-hour period, respectively.

Here, a length of ten days was chosen for $J$ by following Wergen (1987). Figure 1 shows an initialized sea-level pressure difference between the MNMI with and without the climatological tidal modes. This semi-diurnal pattern is quite similar to Ballish et al. (1992) and the amplitude is almost consistent with the report of Chapman and Lindzen (1970) that the amplitude of the most dominant spherical harmonics component of the semi-diurnal surface pressure oscillation is $1.22 \mathrm{hPa}$.

\section{b. Incremental normal-mode initialization}

The INMI technique is thoroughly described in Ballish et al. (1992). The basic procedure of the INMI is almost the same as that of the MNMI. The main difference is in the initialized object that is used for the filtering process. In contrast to the MNMI, the INMI uses an analysis increment, which is an analysis field subtracted by a first guess (i.e., a 6 -h forecast), for the filtering process. First, the time tendencies of a first guess and an analysis field are calculated. Second, the increments are calculated for both the time tendency and pre-initialized value by subtracting the first guess from the analysis field. Third, the time tendency of the fast gravity modes in the increment is modified by passing the same MNMI filtering code. Fi-

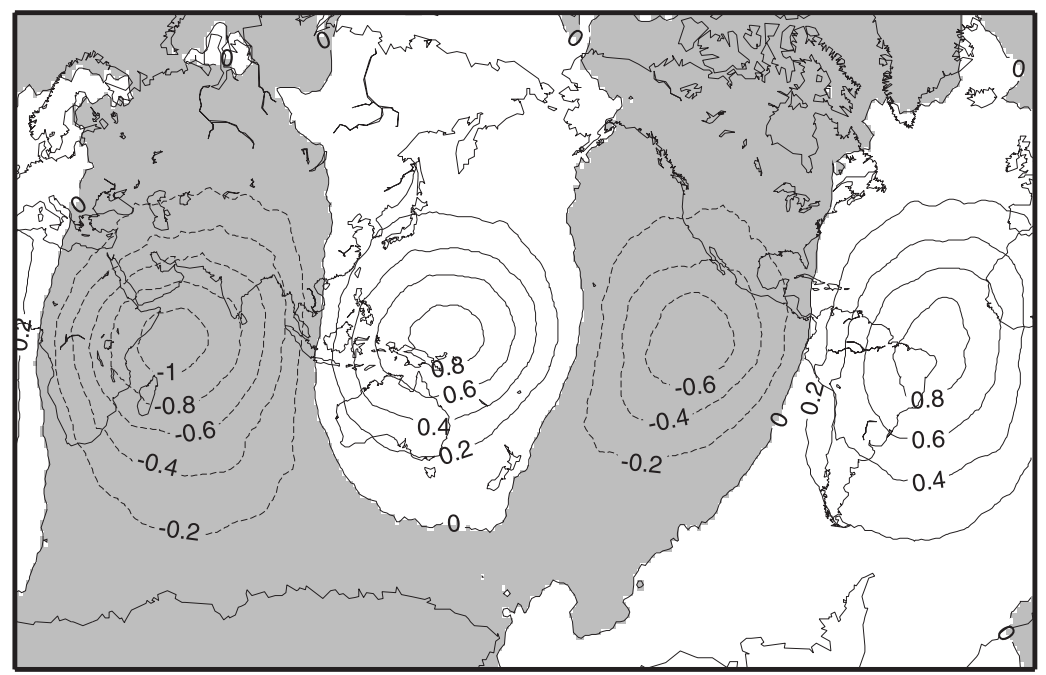

Fig. 1. Initialized sea-level pressure difference between the MNMI with and without climatological tidal modes. The unit is hPa. The date is 12 UTC August 1, 2004. 
nally, the modified increment is added to the first guess. The filtering condition of the INMI is

$$
\frac{\partial G_{A}^{\prime}}{\partial t}=\frac{\partial G_{F}}{\partial t},
$$

where the subscripted $F$ represents the first guess. The filtering procedure can be described as

$$
\left(G_{A}-G_{F}\right)^{\prime}=\left(G_{A}-G_{F}\right)+\frac{\partial G_{A} / \partial t-\partial G_{F} / \partial t}{i \omega} .
$$

When the $G_{F}$ is added to the modified increment in Eq. (8), the modified analysis field is obtained as follows:

$$
G_{A}^{\prime}=G_{A}+\frac{\partial G_{A} / \partial t-\partial G_{F} / \partial t}{i \omega} .
$$

It can be seen from Eq. (7) that the INMI scheme sets the time derivative of the gravity modes to the time derivative of the first guess. An advantage of the INMI over the MNMI is that the INMI can preserve the gravity modes of the first guess, such as the tidal modes, which are unexpectedly removed by the MNMI. Hence, the additional technique of the climatological tidal modes, which is used together with the MNMI, is no longer required for the INMI. On the other hand, the INMI has a risk, namely, that an initialized field contains noise if gravity noise remain in the first guess. Therefore, a first guess without gravity wave noise is necessary for the INMI.

\section{c. Vertical normal-mode initialization}

The fundamental theory of the VNMI with shallow water equations is described in Bourke and McGregor (1983). In this section, an extended application of the VNMI to the JMA/ MRI-AGCM is displayed.

The primitive equations of motion can be vertically discretized for each level $k$ in the following forms:

$$
\begin{aligned}
& \frac{\partial D_{k}}{\partial t}=-\nabla^{2} \Phi_{k}+f \zeta_{k}-\beta U_{k}+N_{D}, \\
& \frac{\partial \Phi_{k}}{\partial t}=-\sum_{l=1}^{K} S_{k l} D_{l}+N_{\Phi}, \\
& \frac{\partial \zeta_{k}}{\partial t}=-f D_{k}-\beta V_{k}+N_{\zeta},
\end{aligned}
$$

where $U$ and $V$ are the eastward and northward components of the horizontal wind velocity, respectively; $\zeta$ is the vorticity; $D$, the divergence; $\Phi$, the geopotential; $f$, the Coriolis parameter; $\beta$, the meridional derivative of the Coriolis parameter; and $K$, the total number of the vertical levels. $S_{k l}(=\mathbf{S})$ denotes a $K \times K$ atmospheric-stability matrix. An $N$ with a subscript indicates non-linear and residual terms. Now, the eigenvalue $\xi^{2}$ and eigenvector matrix $\mathbf{E}\left(=E_{k l}\right)$ of the matrix $\mathbf{S}$ are determined. The matrix $\mathbf{E}$ satisfies the following eigen equation:

$$
\xi_{n}^{2}=\sum_{k, l} E_{n k}^{-1} S_{k l} E_{l n},
$$

where the subscripted $n$ means an eigenvalue number ranging from 1 to $K$, and $E_{k l}^{-1}\left(=\mathbf{E}^{-1}\right)$ is the inverse matrix of the $\mathbf{E}$. The multiplication of each equation (10), (11), and (12) by the matrix $\mathbf{E}^{-1}$ from the left, results in the diagonalization of $\mathbf{S}$, and the vertical-mode decomposition,

$$
\begin{aligned}
& \frac{\partial \tilde{D}_{n}}{\partial t}=-\nabla^{2} \tilde{\Phi}_{n}+f \tilde{\zeta}_{n}-\beta \tilde{U}_{n}+\tilde{N}_{D}, \\
& \frac{\partial \tilde{\Phi}_{n}}{\partial t}=-\xi_{n}^{2} \tilde{D}_{n}+\tilde{N}_{\Phi}, \\
& \frac{\partial \tilde{\zeta}_{n}}{\partial t}=-f \tilde{D}_{n}-\beta \tilde{V}_{n}+\tilde{N}_{\zeta},
\end{aligned}
$$

where

$$
\begin{aligned}
& \left(\tilde{\boldsymbol{D}}_{n}, \tilde{\Phi}_{n}, \tilde{\zeta}_{n}, \tilde{U}_{n}, \tilde{V}_{n}\right) \\
& \quad=\sum_{k=1}^{K} E_{n k}^{-1}\left(D_{k}, \Phi_{k}, \zeta_{k}, U_{k}, V_{k}\right),
\end{aligned}
$$

and

$$
\left(\tilde{N}_{D}, \tilde{N}_{\Phi}, \tilde{N}_{\zeta}\right)=\sum_{k=1}^{K} E_{n k}^{-1}\left(N_{D}, N_{\Phi}, N_{\zeta}\right) .
$$

Here, the eigenvalue $\xi^{2}$ has a unit of geopotential for each vertical mode, which corresponds to $g h$, where $g$ is the acceleration of gravity, and $h$ is the equivalent depth. Figure 2 displays the five largest equivalent depths (i.e., the external mode and the first 4 internal modes), and the associated vertical eigenvectors that are calculated with an isothermal-reference temperature of $300 \mathrm{~K}$ and a surface pressure of $1000 \mathrm{hPa}$. 


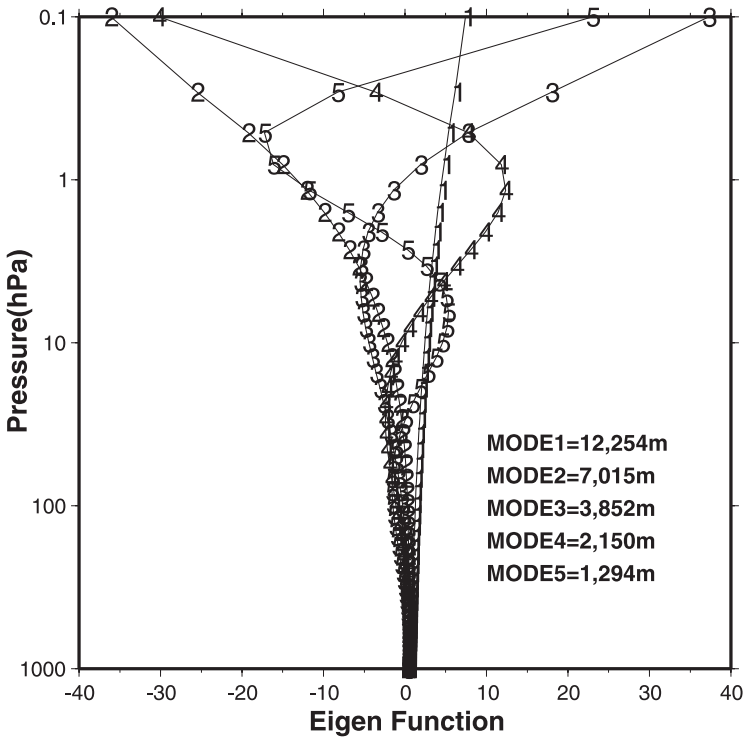

Fig. 2. Five largest equivalent depths and associated vertical eigenvectors. The eigenvectors are ordered by decreasing equivalent depth. The numerical annotation shows the order.

Bourke and McGregor (1983) showed that, if $\beta$ terms are omitted from Eqs. (14) and (16), each vertical mode has a horizontal structure given by individual spherical harmonics. They adopt the omission because their model domain is limited. Although it surely needs careful consideration whether the omission of the $\beta$ terms for a global domain model is allowed or not, we propose it is allowed because only fast gravity modes are modified together with the INMI. It seems reasonable to suppose that very fast gravity modes are not influenced by the Coriolis force. The evidence for this lies in Fig. 3. The diagram shows the eigen-frequency [i.e., $\omega$ in Eq. (1)] of each horizontal gravity mode with a 12,254 m equivalence depth (i.e., the first vertical mode in Fig. 2). (a) and (d) are the diagrams, in which the Coriolis parameter is not fixed, and the $\beta$ terms are not omitted. (a) is for the fast modes whose period is less than 1 hour, and (d) is for slow modes whose period is more than 1 hour. (b) and (e), (c) and (f) are the same as (a) and (d), but for fixed Coriolis parameters at $30^{\circ} \mathrm{N}$ and $60^{\circ} \mathrm{S}$, respectively. The positions of the fast modes [(a), (b), and (c)] turns out to be almost the same. However, the distribution of the slow modes [(d), (e), and (f)] are different from each other. The difference becomes more noticeable as the frequency becomes smaller. This suggests that omitting $\beta$ terms and using the fixed Coriolis parameter do not deteriorate the fast gravity modes but, rather, the slow gravity modes. If the $\beta$ terms are omitted and the Coriolis parameter is fixed for the VNMI, the slow modes should not be changed by the initialization procedure because if they were they would be deformed. Hence, the maximum period (cut-off period) should be set to a relatively small value (e.g., 6 or 9 hours) for the VNMI. Figure 4 also may give a clue for an appropriate cut-off period. The figure shows the projection of the major component of each horizontal eigenvector when the Coriolis parameter is not fixed and $\beta$ terms are not omitted. When the projection of an eigenvector is $100 \%$, it indicates that the eigenvector is parallel to a spherical harmonics component, namely, the mode is not influenced by the Coriolis effect. It can be seen from the figure that the projection for fast modes, whose period is less than 9 hours, is approximately more than $90 \%$. These fast modes seem to be almost the same, even when the Coriolis effect is neglected. However, as the period becomes slow, the projection is decreased and the dispersion of the distribution becomes large. It indicates that careful selection of the cut-off period is necessary when the Coriolis effect is neglected in the VNMI. It is expected that if only fast modes are treated in the initialization procedure, modification pattern is the same whether the Coriolis effect is neglected or not. Moreover, when the INMI is adopted, it is supposed that slow modes are not as dominant in the increment as in the first guess. These slow modes in the first guess are not changed by the INMI, as represented in Eq. (7).

By the above discussion, we venture to suggest that the omission of $\beta$ terms, and using the fixed Coriolis parameter for the global model, are allowed by introducing the INMI and setting a small cut-off period. When the $\beta$ terms are omitted and the Coriolis parameter is fixed, each vertical mode in Eqs. (14), (15), and (16) can be rewritten by introducing spherical harmonics as follows:

$$
\frac{\partial \hat{D}}{\partial t}=\frac{m(m+1)}{a^{2}} \hat{\Phi}+f_{0} \hat{\zeta}+\hat{N}_{D}
$$



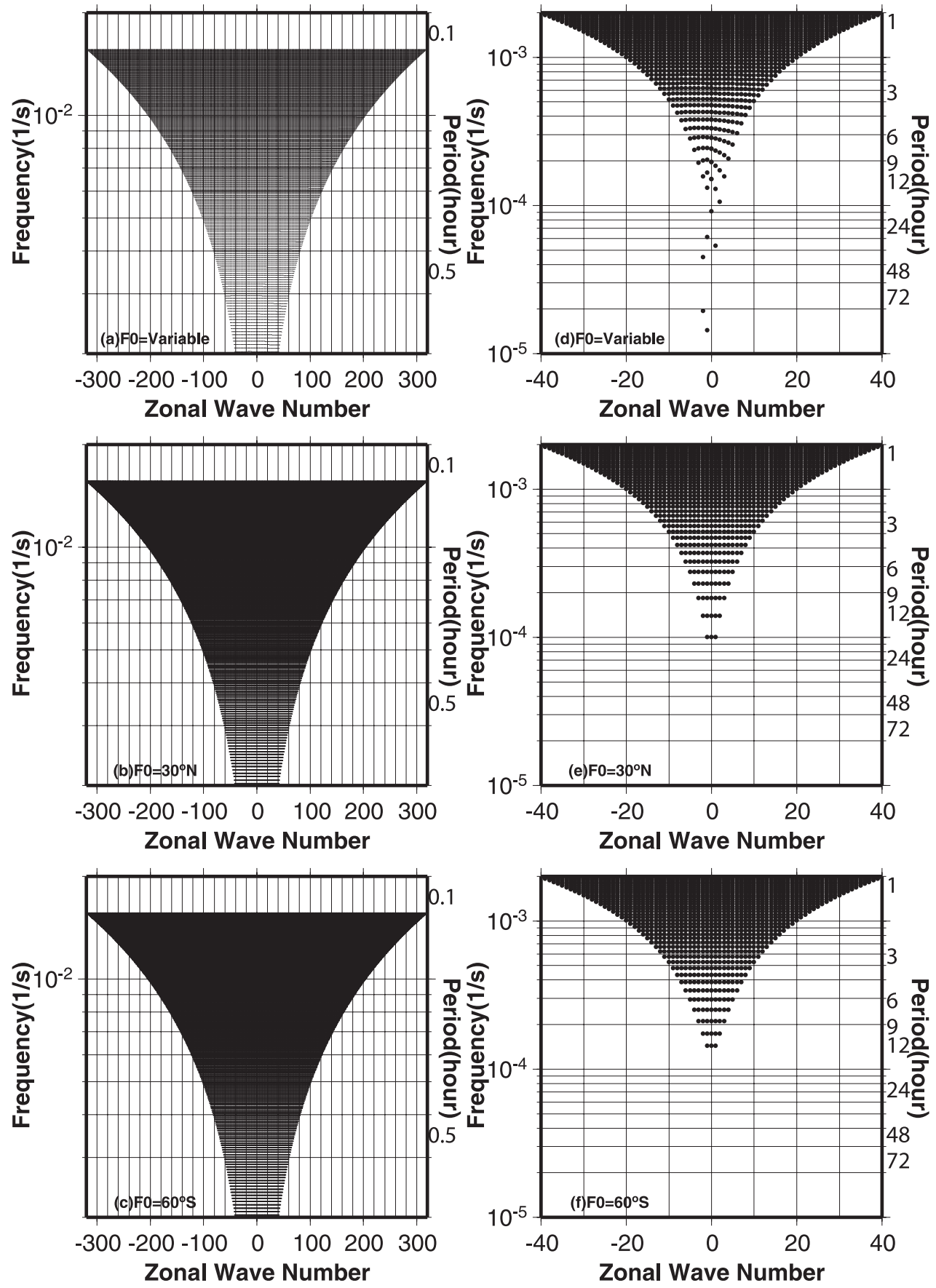

Fig. 3. Eigen-frequency of each horizontal gravity mode with a $12,254 \mathrm{~m}$ equivalent depth. (a) and (d) are the plots, in which the Coriolis parameter is not fixed, and $\beta$ terms are not omitted. The Coriolis parameter is set to $30^{\circ} \mathrm{N}$ in (b) and (e), and $60^{\circ} \mathrm{S}$ in (c) and (f). The frequency [1/s] is given by the left ordinate. The corresponding period [hour] is given by the right ordinate. The zonal wavenumber is given by the abscissa. The model resolution is TL319L40. 


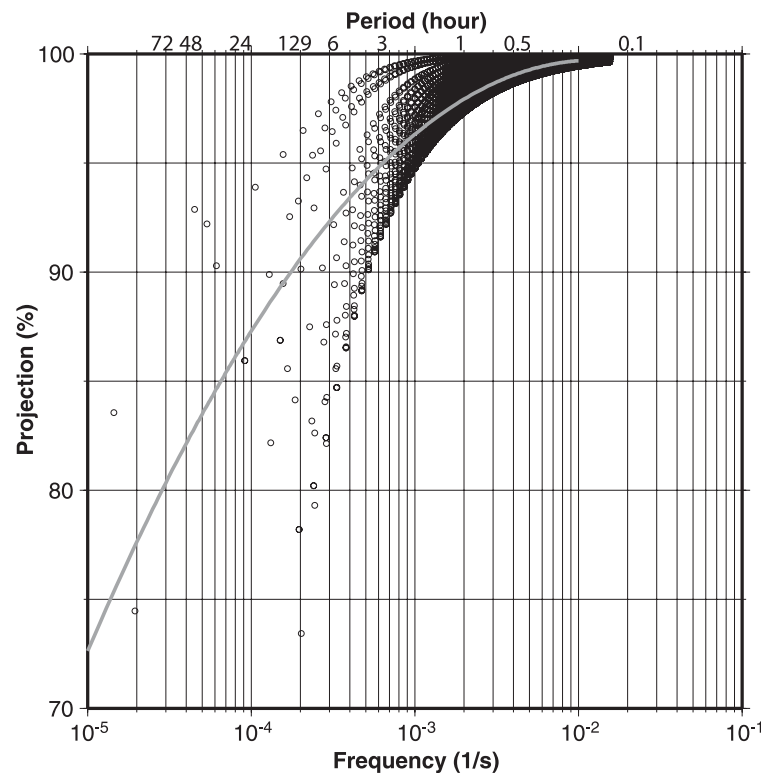

Fig. 4. The projection of the major component of each horizontal eigenvector with a $12,254 \mathrm{~m}$ equivalent depth. The Coriolis parameter is not fixed and $\beta$ terms are not omitted. The frequency $[1 / \mathrm{s}]$ is given by the bottom abscissa. The corresponding period [hour] is given by the upper abscissa. The projection [\%] is given by the ordinate. The gray curve is a simple regression curve $\left(y=-0.532923 \log (x)^{2}-\right.$ $4.672023 \log (x)+89.477771)$.

The model resolution is TL319L40.

$$
\begin{aligned}
& \frac{\partial \hat{\Phi}}{\partial t}=-\xi^{2} \hat{D}+\hat{N}_{\Phi}, \\
& \frac{\partial \hat{\zeta}}{\partial t}=-f_{0} \hat{D}+\hat{N}_{\zeta},
\end{aligned}
$$

where $\left(^{\wedge}\right)$ means the spherical harmonics for each vertical mode; $m$ is the total wavenumber; $a$ is the radius of the earth; $f_{0}$ is the fixed Coriolis parameter at a representative latitude; and the description of the vertical mode $n$ is abbreviated.

Eqs. (19), (20) and (21) can be rewritten in a matrix form as follows:

$$
\frac{\partial}{\partial t}\left(\begin{array}{c}
\frac{\hat{D}}{M} \\
i \frac{\hat{\Phi}}{\xi} \\
i \frac{\hat{\zeta}}{M}
\end{array}\right)=-i \mathbf{G}\left(\begin{array}{c}
\frac{\hat{D}}{M} \\
i \frac{\hat{\Phi}}{\xi} \\
i \frac{\hat{\zeta}}{M}
\end{array}\right)+\left(\begin{array}{c}
\frac{\hat{N}_{D}}{M} \\
i \frac{\hat{N}_{\Phi}}{\zeta} \\
i \frac{\hat{N}_{\zeta}}{M}
\end{array}\right)
$$

where $i$ is the imaginary unit, $M=\sqrt{\frac{m(m+1)}{a^{2}}}$ and

$$
\mathbf{G}=\left(\begin{array}{ccc}
0 & M \xi & f_{0} \\
M \xi & 0 & 0 \\
f_{0} & 0 & 0
\end{array}\right)
$$

Now, the three eigenvalues of the matrix $\mathbf{G}$ are determined as follows:

$$
\begin{aligned}
& \omega_{1}=\sqrt{f_{0}^{2}+(M \xi)^{2}}, \\
& \omega_{2}=-\omega_{1}, \\
& \omega_{3}=0 .
\end{aligned}
$$

Note that the $\omega_{3}(=0)$ corresponds to the degenerated Rossby mode, which is not changed in the initialization process. Furthermore, the normalized eigenvector matrix $\mathbf{B}$ of the matrix $\mathbf{G}$ can be determined as follows:

$$
\mathbf{B}=\frac{1}{\sqrt{2} \omega_{1}}\left(\begin{array}{ccc}
\omega_{1} & \omega_{1} & 0 \\
-M \xi & M \xi & \sqrt{2} f_{0} \\
-f_{0} & f_{0} & -\sqrt{2} M \xi
\end{array}\right) .
$$

In addition, the inverse matrix of $\mathbf{B}$ is

$$
\mathbf{B}^{-1}=\frac{1}{\sqrt{2} \omega_{1}}\left(\begin{array}{ccc}
\omega_{1} & -M \xi & -f_{0} \\
\omega_{1} & M \xi & f_{0} \\
0 & \sqrt{2} f_{0} & -\sqrt{2} M \xi
\end{array}\right) .
$$

The multiplication of Eq. (22) by $\mathbf{B}^{-1}$ from the left side leads to the following equation:

$$
\begin{aligned}
\frac{\partial}{\partial t}\left(\begin{array}{c}
\hat{X}-i \hat{P} \\
\hat{X}+i \hat{P} \\
i \hat{Q}
\end{array}\right)= & -i\left(\begin{array}{ccc}
-\omega_{1} & 0 & 0 \\
0 & \omega_{1} & 0 \\
0 & 0 & 0
\end{array}\right)\left(\begin{array}{c}
\hat{X}-i \hat{P} \\
\hat{X}+i \hat{P} \\
i \hat{Q}
\end{array}\right) \\
& +\left(\begin{array}{c}
\hat{N}_{D}^{\prime} \\
\hat{N}_{\Phi}^{\prime} \\
\hat{N}_{\zeta}^{\prime}
\end{array}\right),
\end{aligned}
$$

where

$$
\begin{aligned}
& \hat{X}=\frac{1}{\sqrt{2} \omega_{1} M} \omega_{1} \hat{D}, \\
& \hat{P}=\frac{1}{\sqrt{2} \omega_{1} M}\left(M^{2} \hat{\Phi}+f_{0} \hat{\zeta}\right) \\
& \hat{Q}=\frac{1}{\omega_{1} \xi}\left(f_{0} \hat{\Phi}-\xi^{2} \hat{\zeta}\right) .
\end{aligned}
$$

Furthermore, the non-linear terms are 


$$
\left(\begin{array}{c}
\hat{N}_{D}^{\prime} \\
\hat{N}_{\Phi}^{\prime} \\
\hat{N}_{\zeta}^{\prime}
\end{array}\right)=\mathbf{B}^{-1}\left(\begin{array}{c}
\frac{\hat{N}_{D}}{M} \\
i \frac{\hat{N}_{\Phi}}{\xi} \\
i \frac{\hat{N}_{\zeta}}{M}
\end{array}\right) .
$$

Now, we focus on the first and second rows of the matrix equation (29),

$$
\begin{gathered}
\frac{\partial}{\partial t}(\hat{X}-i \hat{P})=i \omega_{1}(\hat{X}-i \hat{P})+\hat{N}_{D}^{\prime}, \\
\frac{\partial}{\partial t}(\hat{X}+i \hat{P})=-i \omega_{1}(\hat{X}+i \hat{P})+\hat{N}_{\Phi}^{\prime} .
\end{gathered}
$$

Here, we apply the filtering condition of Eq. (7), which is characterized as setting the time derivative of a gravity mode of an analysis field to that of a first guess. The modified value of a gravity mode for an analysis field $(\hat{X}-i \hat{P})_{A}^{\prime}$ in Eq. (34) can be determined by Eq. (9) as follows:

$$
\begin{aligned}
(\hat{X}-i \hat{P})_{A}^{\prime}= & (\hat{X}-i \hat{P})_{A} \\
& -\frac{1}{i \omega_{1}}\left\{\frac{\partial(\hat{X}-i \hat{P})_{A}}{\partial t}-\frac{\partial(\hat{X}-i \hat{P})_{F}}{\partial t}\right\} \\
= & (\hat{X}-i \hat{P})_{A}-\frac{1}{i \omega_{1}} \frac{\partial(\hat{X}-i \hat{P})_{I}}{\partial t}, \quad(36)
\end{aligned}
$$

where the subscripted $A, F$, and $I$ denote the analysis field, the analysis first guess, and the increment, respectively. $(\hat{X}+i \hat{P})_{A}^{\prime}$ in Eq. (35) can be also determined by Eq. (9) as follows:

$$
\begin{aligned}
(\hat{X}+i \hat{P})_{A}^{\prime}= & (\hat{X}+i \hat{P})_{A} \\
& +\frac{1}{i \omega_{1}}\left\{\frac{\partial(\hat{X}+i \hat{P})_{A}}{\partial t}-\frac{\partial(\hat{X}+i \hat{P})_{F}}{\partial t}\right\} \\
= & (\hat{X}+i \hat{P})_{A}+\frac{1}{i \omega_{1}} \frac{\partial(\hat{X}+i \hat{P})_{I}}{\partial t} .
\end{aligned}
$$

The modifying values of $\hat{X}_{A}$, and $\hat{P}_{A}$, to be added by the filtering condition, can be derived by Eq. (36) and Eq. (37):

$$
\Delta \hat{X}_{A}=\frac{1}{\omega_{1}} \frac{\partial \hat{P}_{I}}{\partial t}
$$

and

$$
\Delta \hat{P}_{A}=-\frac{1}{\omega_{1}} \frac{\partial \hat{X}_{I}}{\partial t} .
$$

The modifying value of $\hat{Q}$ can be determined by focusing on the third row of the matrix equation (29):

$$
\frac{\partial \hat{Q}_{A}^{\prime}}{\partial t}=-i \hat{N}_{\zeta}^{\prime} .
$$

$\hat{N}_{\zeta}^{\prime}$ can be derived by Eq. (28) and Eq. (33),

$$
\hat{N}_{\zeta}^{\prime}=\frac{i}{\omega_{1} \xi}\left(f_{0} \hat{N}_{\Phi}-\xi^{2} \hat{N}_{\zeta}\right)
$$

Eq. (40) can be rewritten using Eq. (20), Eq. (21), Eq. (32), and Eq. (41):

$$
\begin{aligned}
\frac{\partial \hat{\boldsymbol{Q}}_{A}^{\prime}}{\partial t} & =\frac{1}{\omega_{1} \xi}\left(f_{0} \hat{N}_{\Phi}-\xi^{2} \hat{N}_{\zeta}\right) \\
& =\frac{1}{\omega_{1} \xi}\left(f_{0} \frac{\partial \hat{\Phi}}{\partial t}-\xi^{2} \frac{\partial \hat{\zeta}}{\partial t}\right) \\
& =\frac{\partial \hat{\boldsymbol{Q}}_{A}}{\partial t} .
\end{aligned}
$$

Hence, the modifying value of $\hat{Q}$ is zero,

$$
\Delta \hat{\boldsymbol{Q}}_{A}=0 .
$$

The modifying values of $\hat{D}_{A}, \hat{\Phi}_{A}$ and $\hat{\zeta}_{A}$ can be determined by using Eqs. (30), (31), (32), (38), (39), and (43),

$$
\begin{aligned}
& \Delta \hat{D}_{A}=\frac{M^{2}}{\omega_{1}^{2}} \frac{\partial \hat{\Phi}_{I}}{\partial t}+\frac{f_{0}}{\omega_{1}^{2}} \frac{\partial \hat{\zeta}_{I}}{\partial t}, \\
& \Delta \hat{\Phi}_{A}=-\frac{\xi^{2}}{\omega_{1}^{2}} \frac{\partial \hat{D}_{I}}{\partial t}, \\
& \Delta \hat{\zeta}_{A}=-\frac{f_{0}}{\omega_{1}^{2}} \frac{\partial \hat{D}_{I}}{\partial t} .
\end{aligned}
$$

These modifying values are determined iteratively by integrating one-step forward for a few times. In this study, the five fastest vertical modes are modified with 4 iterations, as in the MNMI (see Section 3.a).

There is a remarkable difference between the VNMI and the MNMI, regarding the computational efficiency. In the VNMI, the main computational memory allocation is proportional to the size of the eigenvector matrix, which is seen as $\mathbf{E}$ in Eq. (13). However, the MNMI requires horizontal-mode discretization from Eq. (14) to Eq. (16), because the corresponding matrix $\mathbf{G}$ in Eq. (23) is not symmetric by the $\beta$ terms and the Coriolis parameter $f$. Hence, the MNMI requires a horizontal eigenvector matrix as well. Here, we define $N_{h}$ and $N_{v}$, which are the horizontal and vertical grid numbers of the model, respectively. The size of the eigenvector matrix in the MNMI is $N_{v} \times N_{v}$ for 
Table 1. Size of the required memory allocation of an eigenvector for each normal-mode discretization for JMA/ MRI-AGCM.

\begin{tabular}{|l|c|c|}
\hline Resolution & $\begin{array}{c}\text { Vertical mode } \\
\text { discretization } \\
\text { (KByte) }\end{array}$ & $\begin{array}{c}\text { Horizontal mode } \\
\text { discretization } \\
\text { (MByte) }\end{array}$ \\
\hline T106L40 & 25.00 & 31.60 \\
\hline T213L40 & 25.00 & 251.00 \\
\hline TL319L40 & 25.00 & 837.27 \\
\hline TL511L60 & 56.25 & $3,423.38$ \\
\hline TL959L60 & 56.25 & $22,535.25$ \\
\hline
\end{tabular}

vertical-mode discretization, and $3 N_{h} \times 3 N_{h}$ for horizontal-mode discretization. Table 1 shows the size of the required memory allocation for each normal-mode discretization. The order of memory allocation in practical use for vertical discretization is $O\left(N_{v}^{2}\right)$, whereas that for horizontal discretization is $O\left(N_{h}^{3}\right)$. This shows that the computational-memory resource for the VNMI does not depend on the horizontal resolution, whereas that for the MNMI becomes larger by the horizontal-resolution increase, rather than by the vertical-resolution increase.

\section{Results and discussion}

\section{a. Configuration of experiments}

For a preliminary attempt to implement the new initialization scheme in a super highresolution model, we will show a comparison between the new scheme and the MNMI with a relatively middle-resolution (i.e., TL319L40; horizontal $60 \mathrm{~km}$-mesh, vertical 40 layers) model. Section 4.b displays a difference in the change of an initial field caused by the use of each initialization scheme. The first guess required for the INMI is derived from a 6-hour forecast which was obtained using the JMA global analysis data. In order to assess the impact of introducing the new scheme on forecast skill, Section 4.c shows the result of a mediumrange forecast experiment with data assimilation. The data assimilation system used in the experiment is a low-resolution (T106L40; horizontal $125 \mathrm{~km}$-mesh, vertical 40 layers) version of the operational global data assimilation system of the JMA, (JMA 2002). Figure 5 shows a schematic configuration of the experiment with data assimilation. The assimilation, cycled four times a day $(00,06,12$, and 18 UTC), is a threedimensional variational (3DVar) data assimilation scheme. An incremental method (Courtier et al. 1994) is adopted in the 3DVar to save computational resources. The method calculates analysis increments at a lower resolution (T63L40; horizontal $200 \mathrm{~km}$-mesh, vertical 40 layers), and then adds the increments to the first guess at the original resolution (T106L40). Some of the noise in the analysis field may come from the difference of resolution in the incremental method (e.g., difference in topography). The first guess, which is required for the 3DVar and the INMI, is derived from a 6 -hour forecast. On the other hand, the first guess is not required in the MNMI.

Finally, we will show the performance of the new initialization scheme with the super highresolution (TL959L60) model in Section 4.d. Because we do not have a data assimilation system for such a high resolution, the $20 \mathrm{~km}$ mesh analysis data is created by an interpolation from the $60 \mathrm{~km}$-mesh JMA analysis. The first guess, needed for the INMI, is derived from a 6 -hour forecast. This super highresolution test is conducted on the ES using 30 nodes.

\section{b. Change of the initial states}

As stated in Section 3.c, the VNMI sets the fixed Coriolis parameter, and requires the INMI and a relatively small cut-off period. In order to see how the modification pattern depends on the Coriolis parameter and the cut-off period, a comparison of the modification pattern by changing these parameters is conducted with the middle-resolution model. Figure 6 shows the modification patterns of the sea-level pressure with various parameter settings. The Coriolis parameter is fixed to $60^{\circ} \mathrm{N}$, $30^{\circ} \mathrm{N}, 30^{\circ} \mathrm{S}$, and $60^{\circ} \mathrm{S}$ for the new scheme, whereas it is not fixed for the MNMI. Note that the addition of the climatological tides is not introduced for the MNMI cases here. The cut-off period is set to 6.0 hours, 9.0 hours, and 12.0 hours for both the new scheme and the MNMI. The three top panels [(a), (f), and (k)] are the modification patterns by the MNMI. Figure 7 also shows the uninitialized sea-level pressure. Most of the small-scale modification 


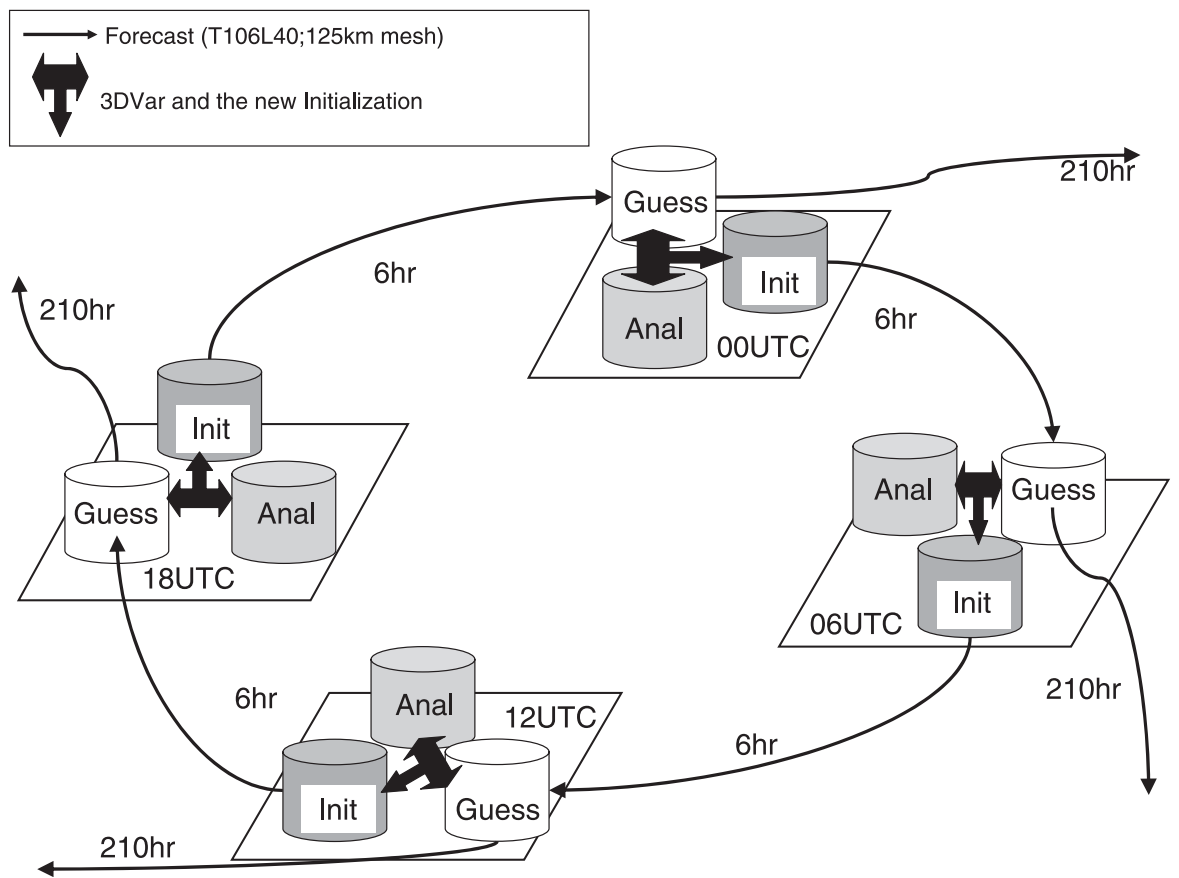

Fig. 5. Configuration of assimilation and forecast experiments. The boxes Anal, Guess, and Init correspond to analysis, first-guess, and initialized data, respectively. The thin arrows are deterministic forecasts, and the thick three-pronged arrows are the 3DVar and the new initializatoin scheme.

pattern caused by the use of the new initialization is the same as that caused by the MNMI. For example, there are two depressions at the south and southwest of Australia. Both initializations increase $0.5-2.0 \mathrm{hPa}$ on those depressions. However, in the case of the 12-hour cutoff period, the new schemes (1)-(o) are quite different from the MNMI of $(\mathrm{k})$. Although the semi-diurnal tidal pattern is filtered out by the MNMI, it is preserved by the new schemes. Moreover, it is noticeable that (1)-(o) differ from each other. This supports the inference that the slow gravity modes are deteriorated by omitting the $\beta$ terms and using the fixed Coriolis parameter in the new scheme. However, when the cut-off period is smaller, the new scheme shows a similar pattern to the MNMI [(g)-(j) and (f) for 9.0 hours and (b)-(e) and (a) for 6.0 hours]. Moreover, the smaller the cut-off period is, the smaller the difference between modifications by the new scheme is. Hence, it is reasonable to suppose that, when the cut-off period is small, the modified value is not dependent upon the Coriolis parameter. Hereafter, the Coriolis parameter is set to $30^{\circ} \mathrm{N}$, and the cut-off period is set to 9.0 hours for the new scheme.

Figure 8 shows a sea-level pressure change caused by the use of the MNMI with the climatological tidal modes. The cut-off period of the MNMI is set to 48 hours as the default setting of conventional use (see Section 3.a). The MNMI with the climatological tidal modes creates a wide-spread modification which has wavenumber one globally. Furthermore, the amplitude of modification by the MNMI is relatively larger than that by the new scheme. It is supposed that the addition of the climatological tidal modes caused this unnatural-modification pattern for the MNMI case. This large-scale modification may diminish the accuracy of forecast.

A comparison of Root Mean Square (RMS) changes over the entire grid caused by the use of initialization is also examined with the middle-resolution model. Figure 9 shows the 

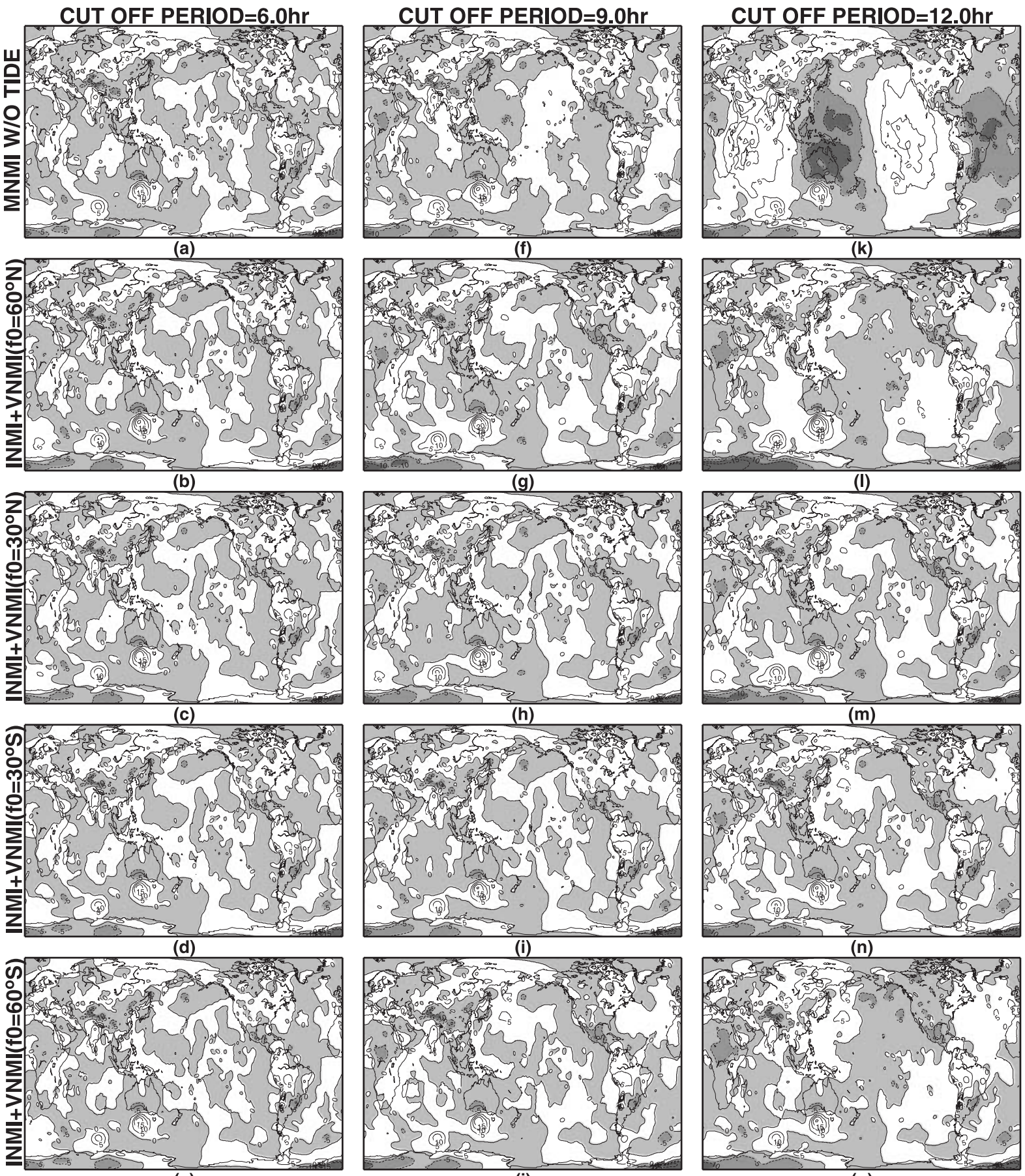

(e)

(j)
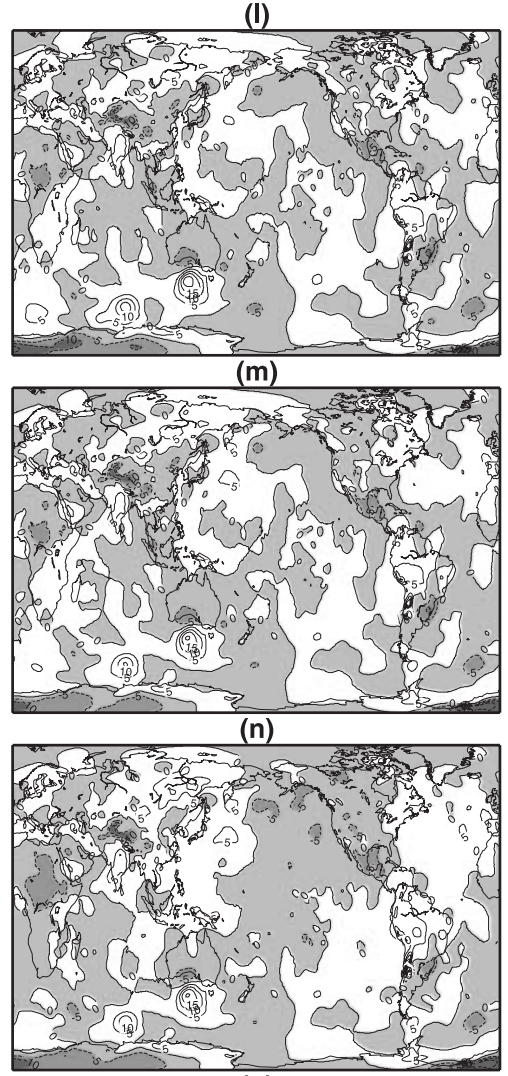

(o)

Fig. 6. Modification patterns of the sea-level pressure (a) by the MNMI without the climatological tidal modes and (b) by the new scheme, in which the Coriolis parameter is fixed as $f_{0}=60^{\circ} \mathrm{N}$. (c), (d), and (e) are the same as (b), but for $f_{0}=30^{\circ} \mathrm{N}, f_{0}=30^{\circ} \mathrm{S}$, and $f_{0}=60^{\circ} \mathrm{S}$, respectively. The cutoff period of (a)-(e) is 6.0 hours. (f) $-(\mathrm{j})$ and (k)-(o) are the same as (a)-(e), but the cut-off period are 9.0 hours and 12.0 hours, respectively. The unit is $0.1 \mathrm{hPa}$. The model resolution is TL319L40. 


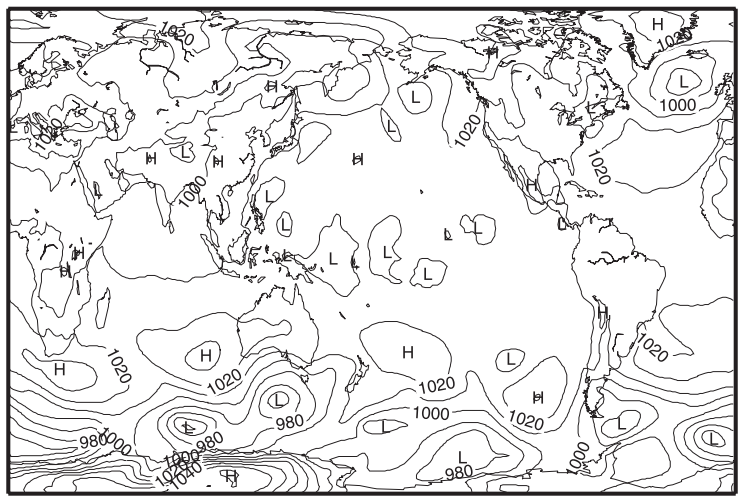

Fig. 7. Uninitialized sea-level pressure [hPa] at 12 UTC JUN 2003. The model resolution is TL319L40.

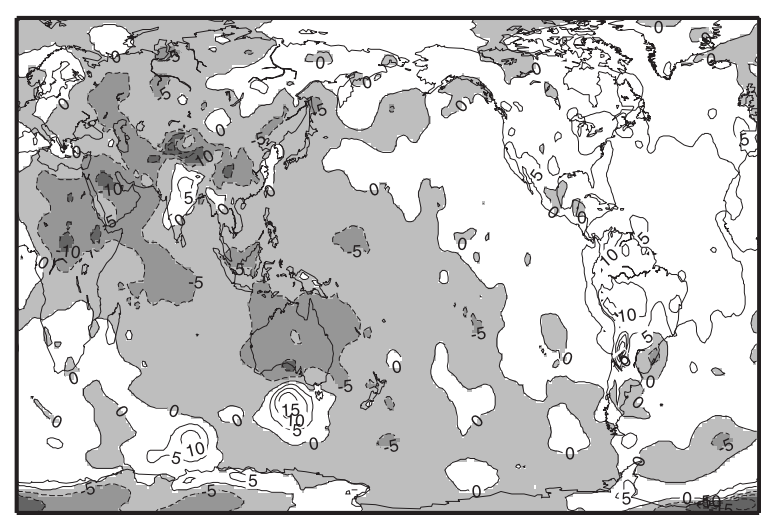

Fig. 8. Changes of the initial sea-level pressure by the MNMI with the climatological tidal modes. The unit is $\mathrm{hPa}$. The contour interval is $0.1 \mathrm{hPa}$.

Fig. 9. Root-mean-square (RMS) changes over the entire grid caused by the use of initialization. (a) Temperature, (b) zonal velocity, and (c) meridional velocity. The cross plots show the RMS by the new scheme. The open-circle plots show the RMS by the MNMI, with the climatological tidal modes. The model resolution is TL319L40.
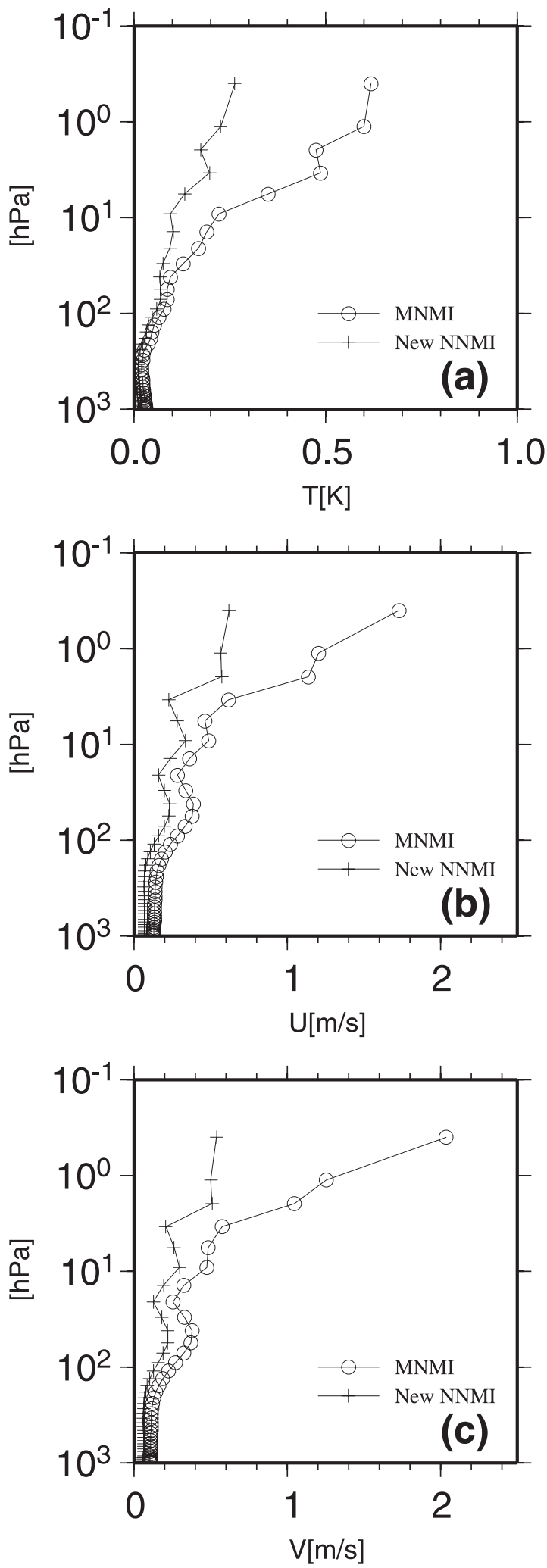

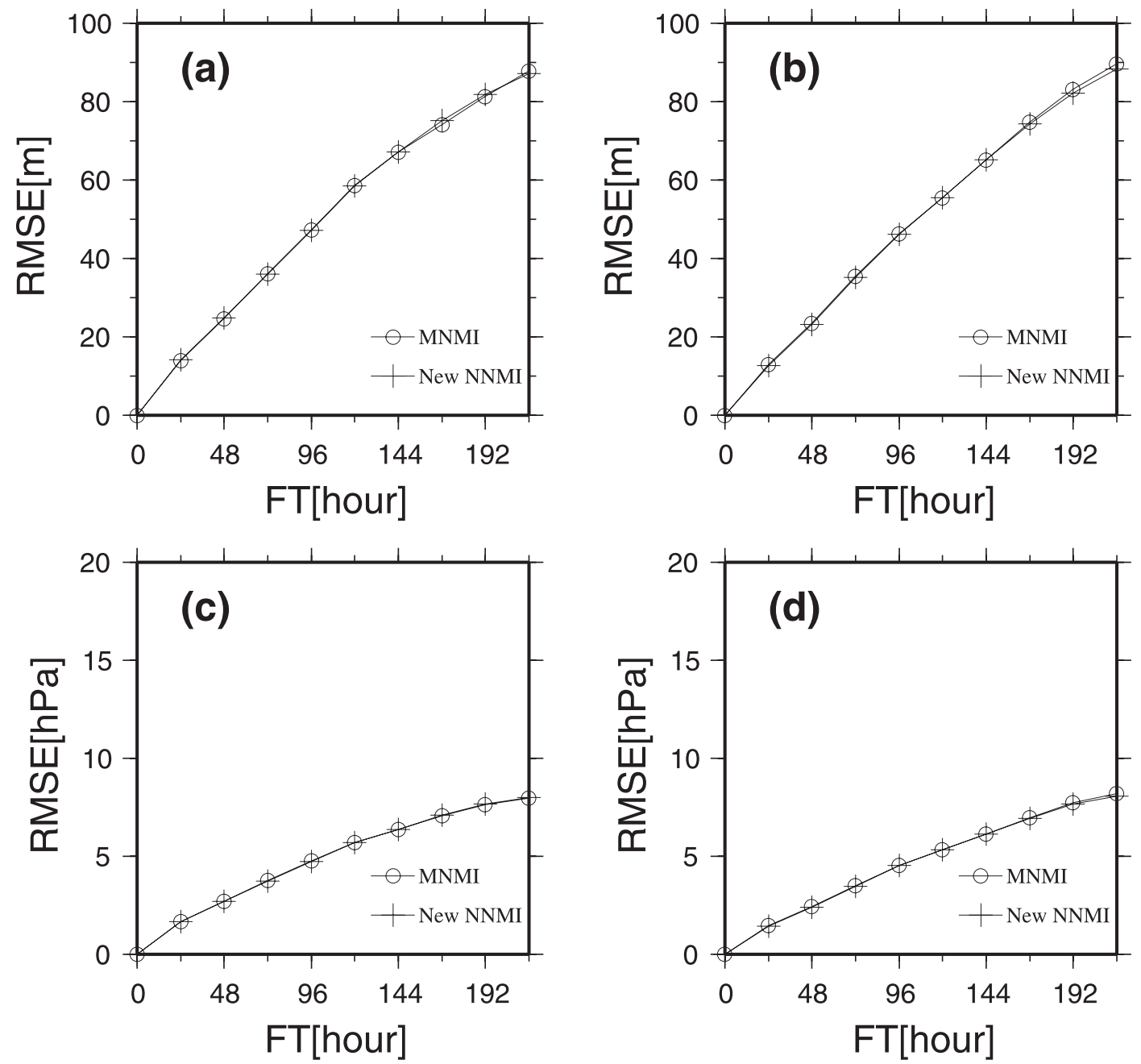

Fig. 10. Root-mean-square error (RMSE) against analysis over the global domain. (a) $500 \mathrm{hPa}$ geopotential height in summer, (b) $500 \mathrm{hPa}$ geopotential height in winter, (c) sea-level pressure in summer, and (d) sea-level pressure in winter. The open-circle plots show the experiment by the MNMI with the climatological tidal modes. The cross plots show the experiment with the new initialization scheme. The summer experiment is for July 2002. The winter experiment is for January 2003.

RMS changes of temperature, zonal velocity, and meridional velocity, caused by the use of each initialization scheme. The RMS of the temperature ranges from 0 to $0.7 \mathrm{~K}$; the winds range from 0 to $2.0 \mathrm{~m} / \mathrm{s}$ for both initialization schemes. The RMS changes of all elements by the MNMI with the climatological tidal modes are larger than those by the new scheme at upper levels. These diagrams show that the new scheme does not cause large variations in the wind, and the temperature from those of the analysis field.

\section{c. Impact on the forecast performance}

In order to investigate the impact of the new scheme on the forecast skill, a forecast experiment with data assimilation is examined for each initialization scheme. Figure 10 shows the Root Mean Square Error (RMSE) of $500 \mathrm{hPa}$ geopotential height and sea surface pressure against analysis over the global domain for the summer (July of 2002) and winter (January of 2003) experiments. Although the new scheme is simple compared with the MNMI, the forecast skill is almost the same. 
MNMI

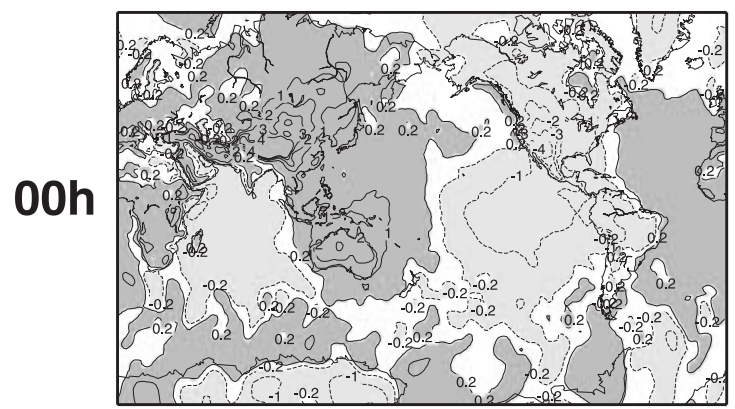

(a)

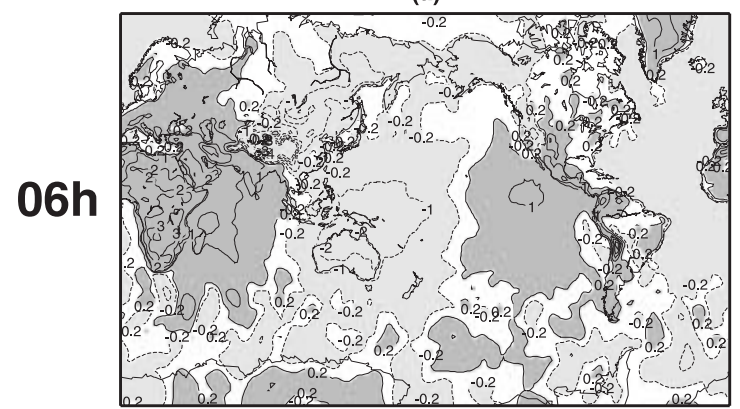

(b)

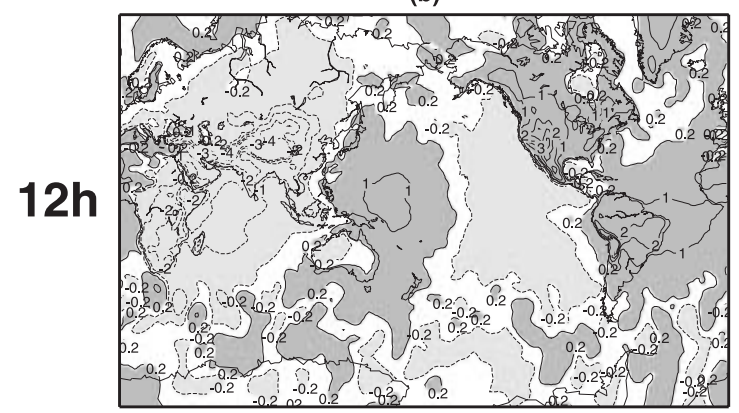

(c)

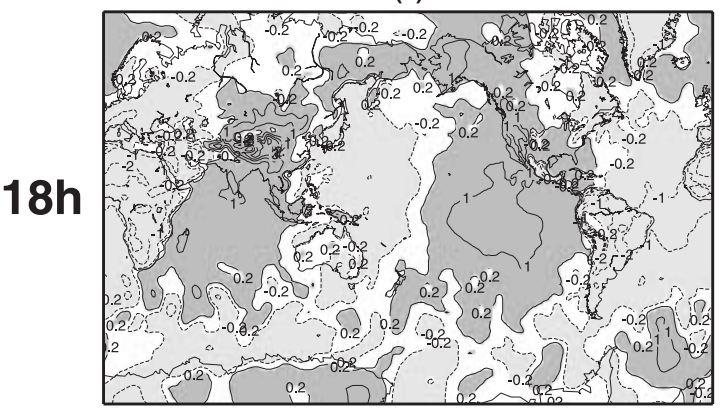

(d)
NEW NNMI

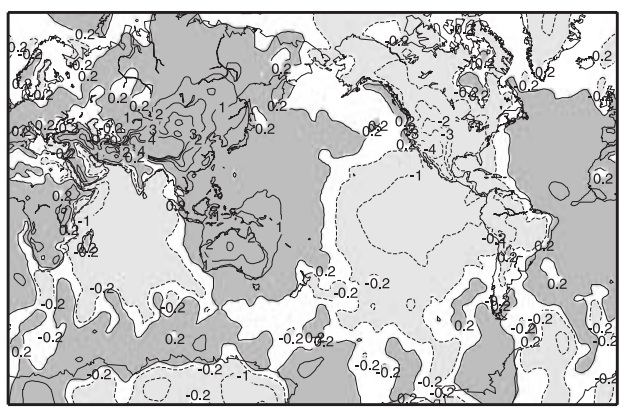

(e)

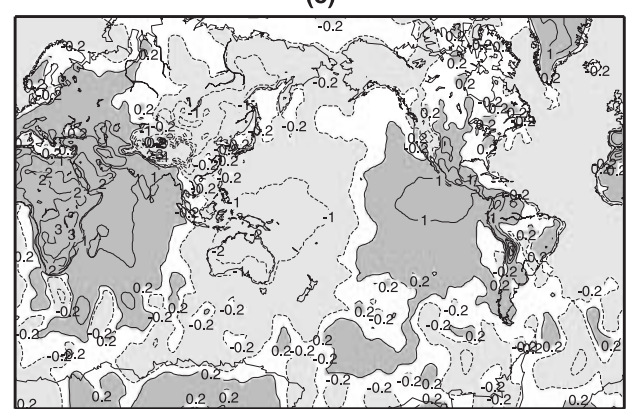

(f)

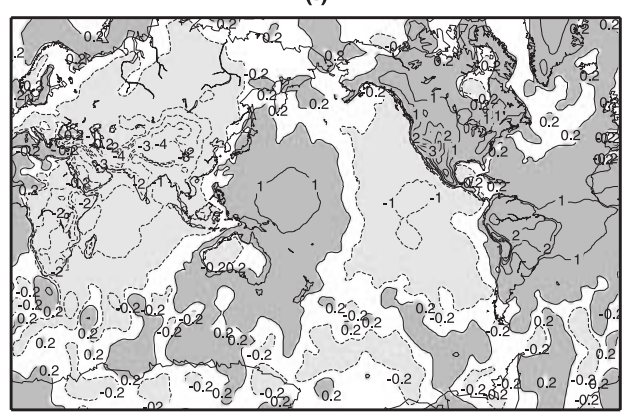

(g)

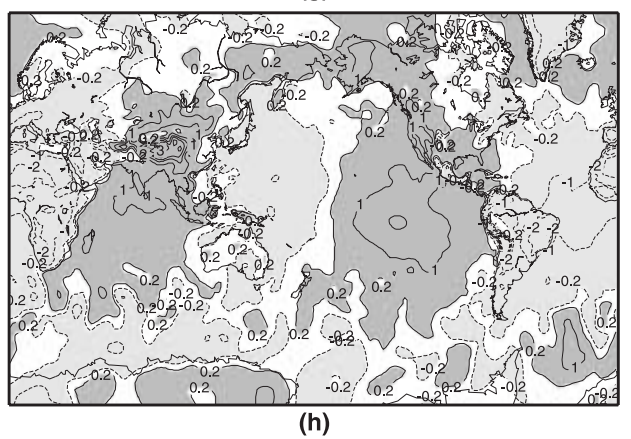

Fig. 11. Sea-level pressure anomaly from one-month averaged analysis field. (a), (b), (c), and (d) are by the MNMI with the climatological tidal modes for 00 UTC, 06 UTC, 12 UTC, and 18 UTC, respectively. (e), (f), (g), and (h) are the same but by the new scheme. Light(dark) shading indicates an anomaly of less(more) than $-0.2 \mathrm{hPa}(0.2 \mathrm{hPa})$. 
During the assimilation experiments, we checked that the analysis field conserves the tidal modes with the new scheme. Figure 11 shows an anomaly of sea-level pressure for four analysis times from a one-month averaged analysis field. It can be seen that there are small differences between the two initialization schemes. The analysis field turns out to conserve the tidal modes with the new scheme.

The fact that the new scheme conserves the tidal waves of analysis fields more accurately than the MNMI is clear when they are compared with the non-initialized forecasts. Figure 12 shows the time evolution of the surface pressure at four grid points with the new scheme, the MNMI, and without initialization. It is obvious that the lines with the MNMI scheme are separated from the other lines in the early forecast hours. This seems to be caused by the addition of climatological tidal modes, as mentioned in Section 3.a. Overall, the new scheme conserves the tidal modes well. This results in a better first guess in the 6-hour assimilation cycle.

\section{d. Removing high-frequency noise with a super high-resolution model}

As stated in Section 1, the VNMI makes it possible to implement initialization in a super high-resolution model with a grid spacing of $20 \mathrm{~km}$, while the MNMI is difficult to implement because of the extremely large memory allocation. Figure 13 shows the time evolution of the surface pressure with the super highresolution version of the JMA/MRI-AGCM. The solid line is the forecast with the new scheme, while the dotted line is the forecast without initialization. In this example, it is clear that the new scheme removes spurious high-frequency noise in the early forecast hours. Furthermore, a tidal mode, with a period of 12 hours, is evident in the equatorial points.

Figure 14 shows the sea-level pressure for the uninitialized field and the initialized field. Most of the noise seem to be caused by the interpolation error when the analysis data of the $20 \mathrm{~km}$-mesh model is created from the $60 \mathrm{~km}$ mesh JMA analysis. The noise, however, are successfully removed by the new scheme. We also tested another case of strong, or compact typhoons. In such a case, the new scheme turns out to remove noise well, and did not destroy the typhoon structure.

The convergence of the new scheme is examined in terms of the sum of the square of the wave tendencies of the gravity wave mode $\left[\mathrm{BAL}=\Sigma\left(\frac{\partial G_{\mathrm{A}}}{\partial t}\right)^{2}\right]$. Figure 15 shows the average BAL of 42 forecasts for each iteration step. This result shows that the new scheme suppresses the gravity-wave growth well by the iteration steps.

\section{Summary}

We have developed an efficient normalmode initialization scheme for a super highresolution AGCM with a grid spacing of $20 \mathrm{~km}$. The new initialization scheme consists of the vertical normal-mode initialization method and the incremental-normal mode initialization method.

In order to compare the new scheme with the conventional MNMI scheme, changes of the initial field were examined with the middle-resolution (TL319L40) version of the JMA/ MRI-AGCM. The deformation by omitting $\beta$ terms and the fixing of Coriolis parameter does not seem to influence the fast gravity modes. When the cut-off period is set small in the new scheme, the modification pattern of the sealevel pressure does not depend upon the Coriolis parameter, and becomes similar to that by the MNMI without the addition of climatological tidal modes. Furthermore, the MNMI with the addition of climatological tidal modes shows an unnatural modification pattern that has a relatively larger amplitude and spreads globally. The RMS changes in the two schemes were also compared. The RMS changes of the temperature and wind by the MNMI are larger than those by the new scheme, particularly at upper levels.

The forecast performance was also compared between the two initialization schemes. The new scheme was comparable with the MNMI, in terms of RMSE of $500 \mathrm{hPa}$ geopotential height and sea-level pressure against analysis. Among the assimilation experiments, the tidal waves created by the model integration are confirmed and are well conserved with the new scheme, mainly due to the INMI. Moreover, the time evolution of the surface pressure in a forecast was improved in the early forecast stage. 

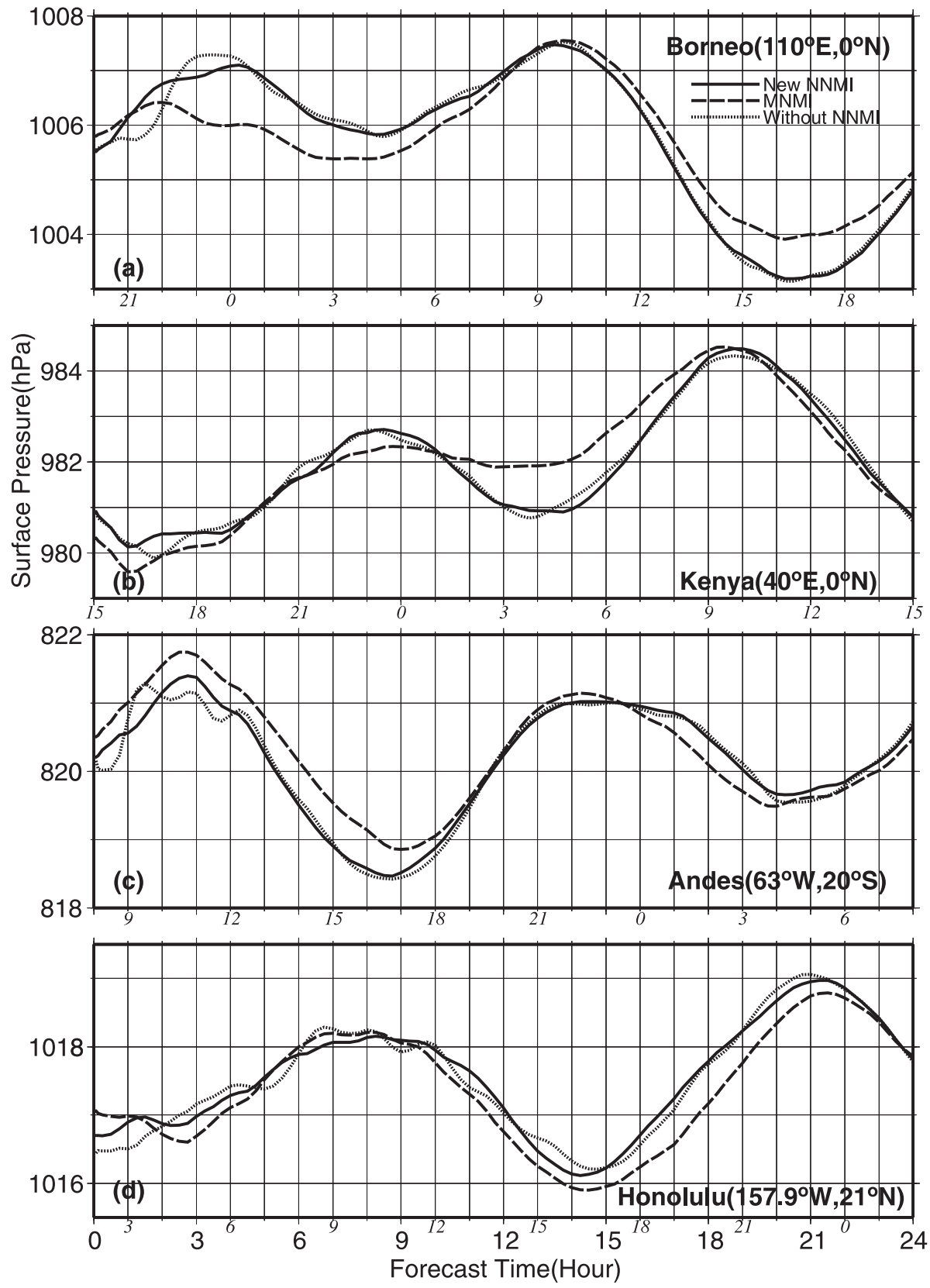

Fig. 12. Surface pressure in $\mathrm{hPa}$ at the grid point of (a) Borneo, (b) Kenya, (c) the Andes, and (d) Honolulu for the first 24 hours. The solid lines are the forecast with the new scheme. The dashed lines are the forecast with the MNMI. The dotted lines are the non-initialized forecast. The initial date is 12 UTC July 20, 2002. The model resolution is T106L40. The numbers in italics on the abscissa show the LST, and those in bold show the forecast time.

Furthermore, the new scheme successfully suppresses spurious high-frequency noise with the super high-resolution AGCM. This result is confirmed by checking the time evolution of the surface pressure at some points, the modification pattern of the sea-level pressure, and the average BAL for each iteration step. 


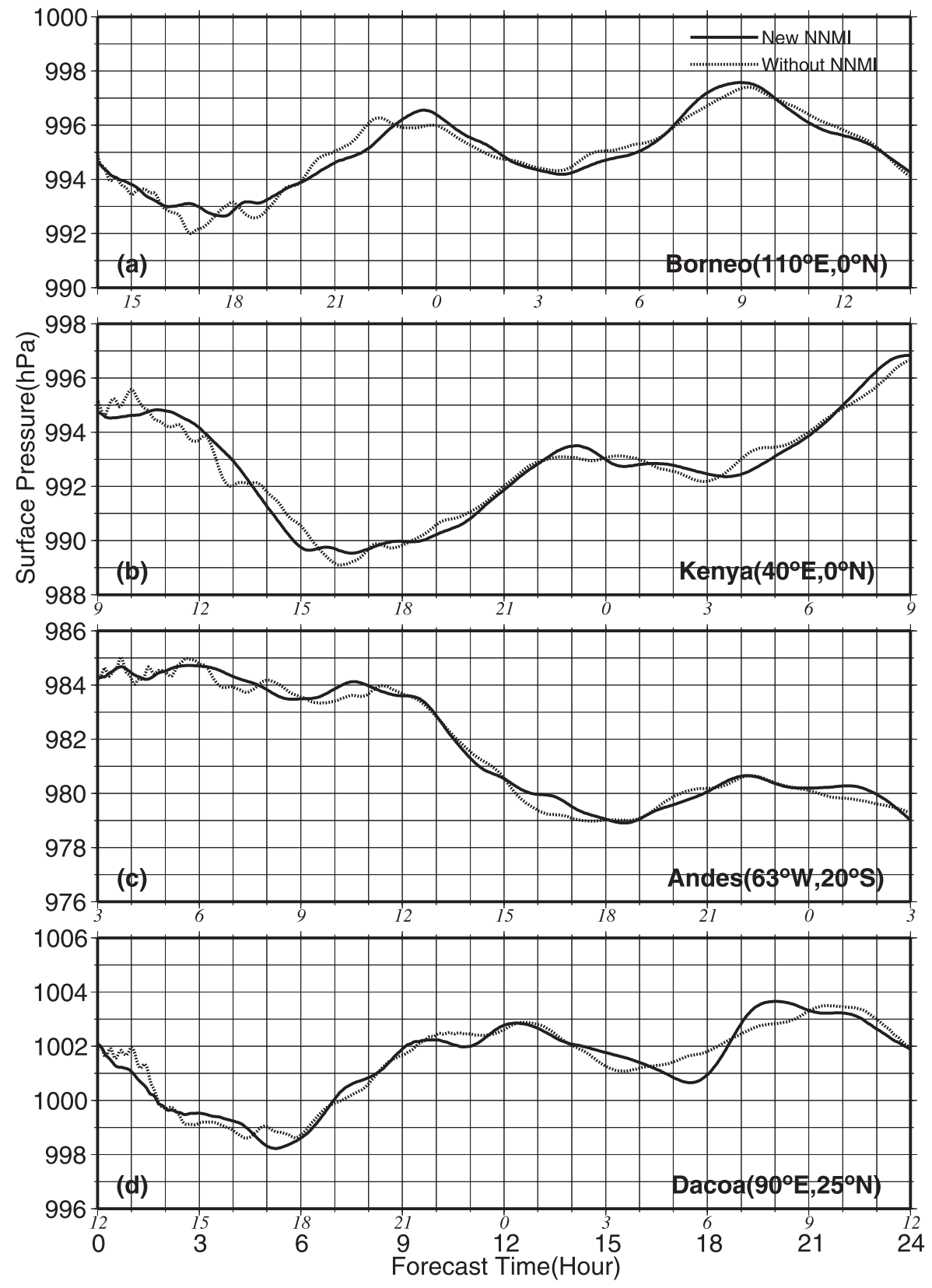

Fig. 13. Surface pressure in hPa for (a) Borneo, (b) Kenya, (c) the Andes, and (d) Dacoa for the first 24 hours. The solid lines are the forecast with the new scheme. The dotted lines are the non-initialized forecast. The initial date is 6 UTC 05 June, 2003. The model resolution is TL959L60. The numbers in italics on the abscissa show the LST, and those in bold show the forecast time. 


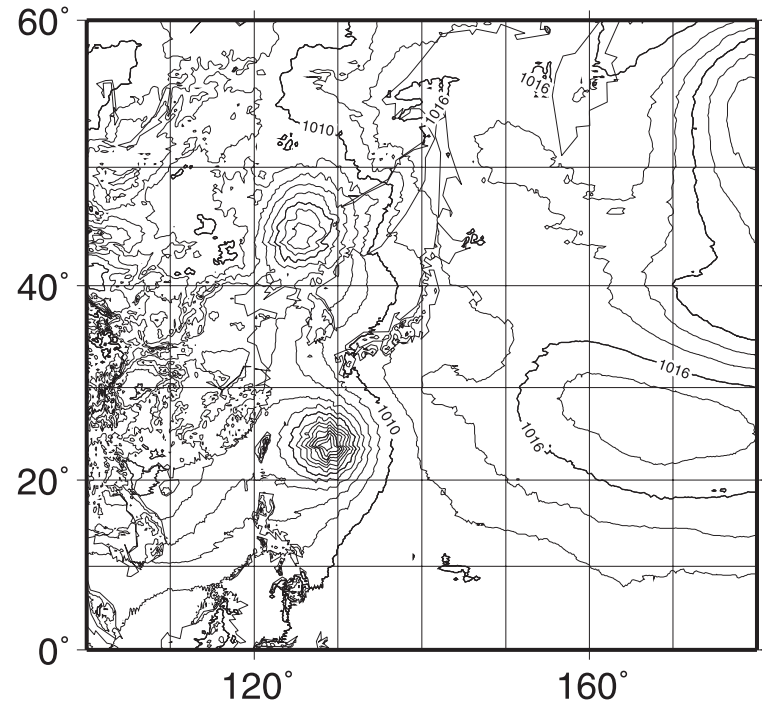

(a)

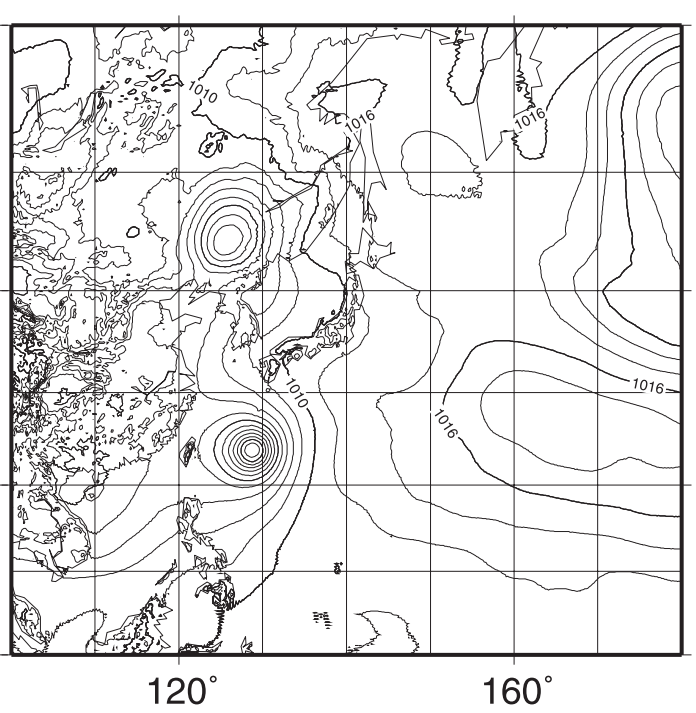

(b)

Fig. 14. Sea level pressure (a) before initialization and (b) after initialization by the new scheme.

The initial date is 12 UTC 06 August, 2002. The contour interval is $2 \mathrm{hPa}$.

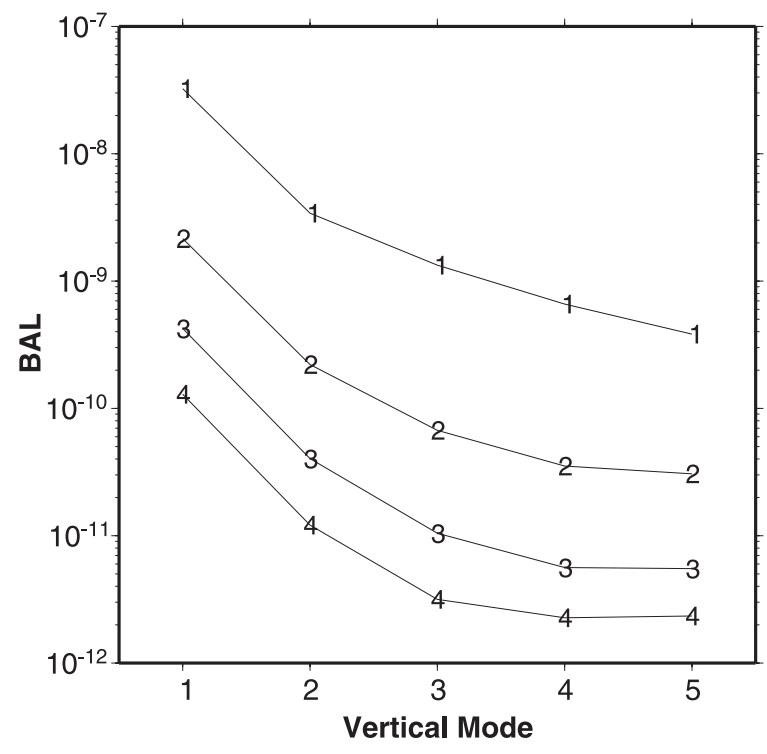

Fig. 15. Change of the BAL for the first five vertical modes from the first to fourth iteration steps. The BAL is calculated from the gravity modes whose periods are shorter than 9 hours. The model resolution is TL959L60.

\section{Acknowledgments}

This work is a part of the "Kyosei Project 4: Development of Super High-Resolution Global and Regional Climate Models" supported by the Ministry of Education, Culture, Sports, Science, and Technology. The authors would like to thank Dr. Tadashi Tsuyuki and Mr. Yousuke Yamazaki for their advice on this paper. The authors would also like to acknowledge the assistance and support of members of the Kyosei Project and the Numerical Prediction Division of JMA. We also wish to thank the late Dr. Hajime Nakamura for his encouragement on this work. Thanks also go to the Earth Simulator Center for providing computational environments.

\section{References}

Arakawa, A. and W.H. Schubert, 1974: Interaction of a cumulus cloud ensemble with the large-scale environment, Part I. J. Atmos. Sci., 31, 674701.

Baer, F., 1977: Adjustment of initial conditions required to suppress gravity oscillations in non- 
linear flows. Beitr. Phys. Atmos., 50, 350-366.

Ballish, B., X. Cao, E. Kalnay, and M. Kanamitsu, 1992: Incremental nonlinear normal-mode initialization. Mon. Wea. Rev., 120, 1723-1734.

Bengtsson, L., M. Botzet, and M. Esch, 1996: Will greenhouse gas-induced warming over the next 50 years lead to higher frequency and greater intensity of hurricanes? Tellus, 48A, $57-73$.

Bourke, W. and J.L. McGregor, 1983: A Nonlinear vertical mode initialization scheme for a limited area prediction model. Mon. Wea. Rev., 111, 2285-2297.

Briegleb, B.P., 1992: Delta-Eddington approximation for solar radiation in the NCAR community climate model. J. Geophys. Res., 97, 76037612 .

Chapman, S. and R.S. Lindzen, 1970: Atmospheric tides, thermal and gravitational. D. Reidel Publishing Company, Dordrecht-Holland, 209 pp.

Coakley, J.A., R.D. Cess, and F.B. Yurevich, 1983: The effect of tropospheric aerosols on the earth's radiation budget: A parameterization for climate models. J. Atmos. Sci., 40, 116-138.

Courtier, P. and O. Talagrand, 1990: Variational assimilation of meteorological observations with the direct and adjoint shallow water equations. Tellus, 42A, 531-549.

Courtier, P., J.-N. Thépaut, and A. Hollingsworth, 1994: A strategy for operational implementation of 4D-Var, using an incremental approach. Quart. J. Roy. Meteor. Soc., 120, 1367-1387.

Daley, R., 1979: The application of non-linear normal mode initialization to an operational forecast model. Atmos.-Ocean., 17, 97-124.

Daley, R., 1980: The development of efficient time integration schemes using model normal modes. Mon. Wea. Rev., 108, 100-110.

Daley, R., 1981: Normal Mode Initialization. Rev. Geophys., 19, 450-468.

Daley, R., 1988: The normal modes of the spherical non-hydrostatic equations with applications to the filtering of acoustic modes. Tellus, 40A, 96-106.

Daley, R., 1991: Atmospheric Data Analysis. Cambridge University Press, 457 pp.

Fillion, L., H.L. Mitchell, H. Ritchie, and A. Staniforth, 1995: The impact of a digital filter finalization technique in a global data assimilation system. Tellus, 47A, 304-323.

Gauthier, P. and J.-N. Thépaut, 2001: Impact of the digital filter as a weak constraint in the preoperational 4DVAR assimilation system of Météo-France. Mon. Wea. Rev., 129, 20892102.
Habata, S., K. Umezawa, M. Yokokawa, and S. Kitawaki, 2004: Hardware system of the Earth Simulator. Parallel Computing, 30, 12871313.

Huang, X.-Y. and P. Lynch, 1993: Diabatic digitalfiltering initialization: Application to the HIRLAM model. Mon. Wea. Rev., 121, 589-603.

Huang, X.-Y. and H. Sundqvist, 1993: Initialization of cloud water content and cloud cover for numerical prediction models. Mon. Wea. Rev., 121, 2719-2726.

Huang, X.-Y., A. Cederskov, and E. Källén, 1994: A comparison between digital filtering initialization and nonlinear normal-mode initialization in a data assimilation system. Mon. Wea. Rev., 122, 1001-1015.

IPCC, 2001: Climate Change 2001: The Scientific Basis. Contribution of Working Group I to the Third Assessment Report of the Intergovernmental Panel on Climate Change. Cambridge University Press, United Kingdom and New York, NY, USA, 881 pp.

Iwasaki, T., S. Yamada, and K. Tada, 1989: A parameterization scheme of orographic gravity wave drag with two different vertical partitionings, part I: Impacts on medium-range forecasts. J. Meteor. Soc. Japan, 67, 11-27.

JMA, 2002: Outline of the operational numerical weather prediction at the Japan Meteorological Agency. $157 \mathrm{pp}$.

Joseph, J.H., W.J. Wiscombe, and J.A. Weinman, 1976: The delta-Eddington approximation for radiative flux transfer. J. Atmos. Sci., 33, 2452-2459.

Kanamitsu, M., K. Tada, T. Kudo, N. Sato, and S. Isa, 1983: Description of the JMA operational spectral model. J. Meteor. Soc. Japan, 61, $812-828$.

Kasahara, A., and J.-H. Qian, 2000: Normal modes of a global nonhydrostatic atmospheric model. Mon. Wea. Rev., 128, 3357-3375.

Katayama, K., H. Yoshimura, and T. Matsumura, 2003: Development of a $20 \mathrm{~km}$ mesh global NWP model on the Earth Simulator. CAS / JSC WGNE Research Activities in Atmospheric and Oceanic Modeling, 33, 0311-0312.

Katayama, K., H. Yoshimura, and T. Matsumura, 2004: Development of a $20 \mathrm{~km}$ mesh global NWP model on the Earth Simulator, CAS / JSC WGNE Research Activities in Atmospheric and Oceanic Modeling, 34, 0311-0312.

Kudo, T., 1982: Normal mode initialization for the spectral model. Denshikeisanshitsu houkoku, Forecast division, JMA, 28, 91-98 (in Japanese).

Kudo, T., 1984: Normal mode initialization for the 
global model. Denshikeisanshitsu houkoku, Forecast division, JMA, 30, 20-22 (in Japanese).

Kusunoki, S., J. Yoshimura, H. Yoshimura, A. Noda, K. Oouchi, and R. Mizuta, 2006: Change of Baiu rain band in global warming projection by an atmospheric general circulation model with a $20-\mathrm{km}$ grid size. J. Meteor. Soc. Japan, 84, 581-611.

Lynch, P. and X.-Y. Huang, 1992: Initialization of the HIRLAM model using a digital filter. Mon. Wea. Rev., 120, 1019-1034.

Lynch, P., D. Giard, and V. Ivanovici, 1997: Improving the efficiency of a digital filtering scheme for diabatic initialization. Mon. Wea. Rev., 125, 1976-1982.

Machenhauer, B., 1977: On the dynamics of gravity oscillations in a shallow water model, with applications to normal mode initialization. Beitr. Phys. Atmos., 50, 253-271.

Mellor, G.L. and T. Yamada, 1974: A hierarchy of turbulence closure models for planetary boundary layers. J. Atmos. Sci., 31, 1791-1806.

Mizuta, R., K. Oouchi, H. Yoshimura, A. Noda, K. Katayama, S. Yukimoto, M. Hosaka, S. Kusunoki, H. Kawai, and M. Nakagawa, 2006: 20km-mesh global climate simulations using JMA-GSM model-mean climate states-. J. Meteor. Soc. Japan, 84, 165-185.

Oouchi, K., J. Yoshimura, H. Yoshimura, R. Mizuta, S. Kusunoki, and A. Noda, 2006: Tropical cyclone climatology in a global-warming climate as simulated in a $20 \mathrm{~km}$-mesh global atmospheric model: Frequency and wind intensity analyses. J. Meteor. Soc. Japan, 84, 259-276.

Palmer, T.N., G.J. Schutts, and R. Swinbank, 1986: Alleviation of a systematic westerly bias in general circulation and numerical weather prediction models through an orographic gravity wave drag parameterization. Quart. J. Roy. Meteor. Soc., 112, 1001-1039.

Polavarapu, S., M. Tanguay, and L. Fillion, 2000: Four-dimensional variational data assimila- tion with digital filter initialization. Mon. Wea. Rev., 128, 2491-2510.

Sato, N., P.J. Sellers, D.A. Randall, E.K. Schneider, J. Shukla, J.L. Kinter III, Y.-T. Hou, and E. Albertazzi, 1989: Effects of implementing the simple biosphere model ( $\mathrm{SiB}$ ) in a general circulation model. J. Atmos. Sci., 46, 2257-2282.

Sellers, P.J., Y. Mintz, Y.C. Sud, and A. Dalcher, 1986: A simple biosphere model ( $\mathrm{SiB}$ ) for use within general circulation models. J. Atmos. Sci., 43, 505-531.

Simmons, A.J. and D.M. Burridge, 1981: An energy and angular-momentum conserving vertical finite-difference scheme and hybrid vertical coordinates. Mon. Wea. Rev., 109, 758-766.

Smith, R.N.B., 1990: A scheme for predicting layer clouds and their water content in a general circulation model. Quart. J. Roy. Meteor. Soc., 116, 435-460.

Sommeria, G. and J.W. Deardorff, 1977: Subgridscale condensation in models of nonprecipitating clouds. J. Atmos. Sci., 34, 344-355.

Temperton, C. and D.L. Williamson, 1981: Normal mode initialization for a multilevel grid-point model. Part I: Linear aspects. Mon. Wea. Rev., 109, 729-743.

Wergen, W., 1987: Diabatic nonlinear normal mode initialization for a spectral model with a hybrid vertical coordinate. ECMWF. Techn. Rep., 59, $83 \mathrm{pp}$.

Williamson, D.L. and C. Temperton, 1981: Normal mode initialization for a multilevel grid-point model. Part II: Non-linear aspects. Mon. Wea. Rev., 109, 744-757.

Wurtele, M.G., R.D. Sharman, and T.L. Keller, 1987: Analysis and simulations of a tropospherestratosphere gravity wave model. Part I. J. Atmos. Sci., 44, 3269-3281.

Yoshimura, H. and T. Matsumura, 2003: A semiLagrangian scheme conservative in the vertical direction. CAS / JSC WGNE Research Activities in Atmospheric and Oceanic Modeling, 33, 0319-0320. 